\title{
THE MULTI-THERMAL AND MULTI-STRANDED NATURE OF CORONAL RAIN
}

\author{
P. Antolin ${ }^{1}$, G. Vissers ${ }^{2}$, T. M. D. Pereira ${ }^{2}$, L. Rouppe van der Voort ${ }^{2}$, and E. Scullion ${ }^{3}$ \\ ${ }^{1}$ National Astronomical Observatory of Japan, Osawa, Mitaka, Tokyo 181-8588, Japan; patrick.antolin@ nao.ac.jp \\ ${ }^{2}$ Institute of Theoretical Astrophysics, University of Oslo, P.O. Box 1029, Blindern, NO-0315 Oslo, Norway \\ ${ }^{3}$ Trinity College Dublin, College Green, Dublin 2, Ireland \\ Received 2015 February 2; accepted 2015 April 16; published 2015 June 9
}

\begin{abstract}
We analyze coordinated observations of coronal rain in loops, spanning chromospheric, transition region (TR), and coronal temperatures with sub-arcsecond spatial resolution. Coronal rain is found to be a highly multithermal phenomenon with a high degree of co-spatiality in the multi-wavelength emission. EUV darkening and quasiperiodic intensity variations are found to be strongly correlated with coronal rain showers. Progressive cooling of coronal rain is observed, leading to a height dependence of the emission. A fast-slow two-step catastrophic cooling progression is found, which may reflect the transition to optically thick plasma states. The intermittent and clumpy appearance of coronal rain at coronal heights becomes more continuous and persistent at chromospheric heights just before impact, mainly due to a funnel effect from the observed expansion of the magnetic field. Strong density inhomogeneities of $0.2-0 .{ }^{\prime \prime} 5$ are found, in which a transition from temperatures of $10^{5}$ to $10^{4} \mathrm{~K}$ occurs. The $0 .{ }^{\prime \prime} 2-$ 0 ." 8 width of the distribution of coronal rain is found to be independent of temperature. The sharp increase in the number of clumps at the coolest temperatures, especially at higher resolution, suggests that the bulk distribution of the rain remains undetected. Rain clumps appear organized in strands in both chromospheric and TR temperatures. We further find structure reminiscent of the magnetohydrodynamic (MHD) thermal mode (also known as entropy mode), thereby suggesting an important role of thermal instability in shaping the basic loop substructure. Rain core densities are estimated to vary between $2 \times 10^{10}$ and $2.5 \times 10^{11} \mathrm{~cm}^{-3}$, leading to significant downward mass fluxes per loop of $1-5 \times 10^{9} \mathrm{~g} \mathrm{~s}^{-1}$, thus suggesting a major role in the chromosphere-corona mass cycle.
\end{abstract}

Key words: instabilities - magnetohydrodynamics (MHD) - Sun: activity - Sun: corona -

Sun: filaments, prominences - waves

Supporting material: animations

\section{INTRODUCTION}

The solar corona, the outermost layer of the solar atmosphere, has an average temperature above a million degrees, a fact that has puzzled astrophysicists for decades. The processes responsible for this heating are still unknown, mostly due to the very short time and spatial scales on which they operate. However, not everything in the corona is hot. Structures at chromospheric temperatures are clearly present in the solar corona. Evidence for this has been found for more than a century, since the first detection of prominences in the solar atmosphere (Secchi 1875). In the last $40 \mathrm{yr}$, especially in the last few years with the advent of high-resolution instruments, smaller chromospheric structures with a more pervasive character have been shown to occur intermittently in the corona above active regions (Kawaguchi 1970; Leroy 1972; Schrijver 2001; Antolin \& Rouppe van der Voort 2012; Antolin et al. 2012; Ahn et al. 2014). This material is known as coronal rain: partially ionized, dense clumpy structures catastrophically cooling in the corona and accreting toward the solar surface. Its occurrence is considered as the direct observational consequence of the thermal instability mechanism, in which radiative losses locally overcome the heating processes.

The presence of prominences in the corona and especially the recent detection of coronal rain with a more pervasive character entails fundamental aspects of the interaction of plasmas and fields in low $\beta$ environment in which thermal instability plays a central role. This mechanism is intrinsically linked to the characteristic cooling aspects of the heating mechanisms and provides far less strict detection possibilities due to the longer timescales and an enormous gain in spatial resolution when observing in wavelengths associated with cool emission. The study of coronal heating through associated cooling therefore provides unique advantages.

A big unknown in the physics of the solar corona is what percentage of coronal plasma is thermally unstable. This is also of significant importance for stellar coronae, since high-speed accretion of chromospheric plasma can produce characteristic profiles in their UV and optical range spectra, both from the falling plasma and from the impact into the lower atmosphere (Ayres \& France 2010; Reale et al. 2013, 2014). Based on numerical simulations, such conditions are expected if the heating is mainly concentrated toward the footpoints (Antiochos et al. 1999; Karpen et al. 2001; Luna et al. 2012), a condition often reported in active regions, and are to a lesser extent related to the temporal character of the heating, i.e., whether it is impulsive or steady (Müller et al. 2003, 2004). Forward modeling of numerical results and comparison with EUV light curves obtained in observations have helped in answering this question, but a debate exists on whether the heating requirements setting loops in a thermal non-equilibrium state fully match the general observed characteristics of active region loops (Mok et al. 2008; Klimchuk et al. 2010; Peter et al. 2012; Lionello et al. 2013; Klimchuk 2014). Coronal rain observations can help answer this question by estimating the coronal volume and mass flux involved in these events. A first attempt at this was performed by Schrijver (2001) using channels from Transition and Coronal Explorer (TRACE), which led to an occurrence interval of several days for one 
active region loop to undergo catastrophic cooling. Based on $\mathrm{H} \alpha$ data from the CRisp Imaging SpectroPolarimeter (CRISP) instrument at the Swedish 1-m Solar Telescope (SST), Antolin \& Rouppe van der Voort (2012, hereafter Paper I) reduced the occurrence frequency to half a day or less, and estimated the coronal rain mass flux to be about one-third of that uploaded into the corona from spicules, thus stressing an important role in the chromosphere-corona mass cycle.

Paper I predicted that most of the rain material may pass undetected in coarse resolution instruments due to average clump $^{4}$ widths of $300 \mathrm{~km}$ (and lengths of $700 \mathrm{~km}$ or so). Condensations were reported to be clustered in space into groups of correlated events, which indicates that the properties of coronal heating can be correlated over a significant spatial scale and that individual strands (the theoretical internal components of coronal loops) are not necessarily uncorrelated in their evolution. However, while most condensations were found to be correlated in time, only a few events, termed "showers" in Paper I, were found to occur close enough, which would produce large enough EUV absorption detectable with the coarser resolution of coronal instruments. Still, the limit cycles that thermally unstable loops experience, predicted from numerical simulations, should be visible observationally as periodic variations of the EUV intensity (which would then be correlated to the appearance of coronal rain). Such behavior has recently been reported and seems to fit well into the thermal non-equilibrium scenario (Froment et al. 2015).

Since it is chromospheric (cool) plasma occurring in the corona, coronal rain constitutes the highest resolution window into the substructure of loops, and provides a tool for revealing the global magnetic field topology. First evidence for this was provided in Paper I, where the tracking of rain over a decaying active region allowed us to calculate the fall angle of the clumps, and therefore to reveal the large-scale morphology of the magnetic field and distinguish several loop families by tracing the clumps along the legs. The coronal rain condensations were reported to be organized locally as strand-like structure with average widths around $300 \mathrm{~km}$ and lengths around $700 \mathrm{~km}$, with nonetheless broad distributions down to 100 and $200 \mathrm{~km}$, and up to 700 and $2400 \mathrm{~km}$, respectively, for widths and lengths. The multi-strand substructure of loops was also reported in on-disk observations of coronal rain with CRISP in Antolin et al. (2012) and more recently in Scullion et al. (2014). The latter work extends such findings to the coronal rain produced in post-flare loops and to the apexes of loops. In that work the distribution of widths peaks at $100 \mathrm{~km}$, clearly indicating that the bulk of the distribution is unresolved due to the lack of resolution. Interestingly, the lengths of some of the observed condensations were reported to span up to $26,000 \mathrm{~km}$, further underlining the global coronal magnetic field tracing possibilities offered by coronal rain.

The strand-like structure revealed by coronal rain along loops appears similar to the thread-like structure observed in prominences of certain types (e.g., active region prominences, Okamoto et al. 2007). A relevant question is therefore whether such structure is strongly dependent on thermal instability, or whether such structure also exists at coronal temperatures under equilibrium conditions, in accordance with the debated multistrand scenario in loops (Klimchuk 2006; Brooks et al. 2012, 2013; Peter et al. 2013). The loss of pressure

\footnotetext{
4 Also referred to as "blobs".
}

produced by thermal instability produces local accumulation of material leading to condensations. Given a thermally unstable loop, the continuous occurrence in the same spot of thermal instability, combined with the produced strong flows (or other possible siphon flows) in a low $\beta$ environment such as the corona can easily lead to strand-like structure, as shown in multidimensional MHD simulations (Fang et al. 2013). In this paper we show that even in this scenario it is reasonable to expect that such strand-like structure is not locally confined to the thermally unstable regions (and thus only confined to the low-temperature plasma in the loop), but extends along the entire loop and therefore to the hot coronal plasma as well. Therefore, even if a loop does not tend to be originally multistranded, it is possible that such organization is attained due to thermal instability and maintained thereafter. Such a scenario, for which we provide support in this paper (also theoretically supported by Low et al. 2012b), has not been considered before and may significantly impact on the evolution of the coronal loop.

It can therefore be expected that a multithermal structure accompanies a multi-strand organization of the plasma in thermally unstable loops. This is shown in Scullion et al. (2014), where loops observed in the coronal filters of AIA are co-spatial with the $\mathrm{H} \alpha$ condensations. The lack of resolution of AIA unfortunately does not allow us to see the details and spatial scales at which such multithermality can exist. In this work, with the help of the high resolution of the Interface Region Imaging Spectrograph (IRIS), we extend this result.

We present two data sets that combine multi-wavelength instruments spanning chromospheric, transition region (TR) and coronal temperatures. SST/CRISP, Hinode/SOT, IRIS/SlitJaw Imaging (SJI) and Solar Dynamics Observatory (SDO)/ AIA are used to reveal the multi-strand and multithermal aspects of coronal rain at unprecedented detail. The paper is organized as follows. In Section 2 the observations are presented. In Sections 3 and 4 the temperature and morphology of the observed condensations are presented, respectively. Results are discussed in Section5 and conclusions are given in Section 6.

\section{OBSERVATIONS}

\subsection{Data Processing}

In this work we analyze two different data sets corresponding to solar limb observations above active regions. The first data set combines data from SST (Scharmer et al. 2003a) with the CRISP spectropolarimeter and the AIA instrument (Lemen et al. 2011) of $S D O$, dates from 26 June 2010 from 10:03 UT to 11:40 UT, and was centered on AR 11084 at $[x, y]=$ $[-875,-319]$. The second data set, which combines the SOT telescope on board Hinode (Tsuneta et al. 2008), the SJI instrument of IRIS (de Pontieu et al. 2014) and SDO/AIA, dates from 2013 November 29 from 22:30 UT to 23:30 UT and was focused on AR 11903. IRIS/SJI was centered at $[x, y]=[944,-264]$ fully containing the Hinode/SOT field of view (FOV), centered at $[x, y]=[959,-220]$. The context images for these coordinated observations are presented in Figures 1 and 2, where different colors denote different wavelengths, and the different FOV of each instrument can be compared.

We mainly focused on the SDO/AIA filters 304,171 , and 193, corresponding to a range of TR to coronal temperatures 


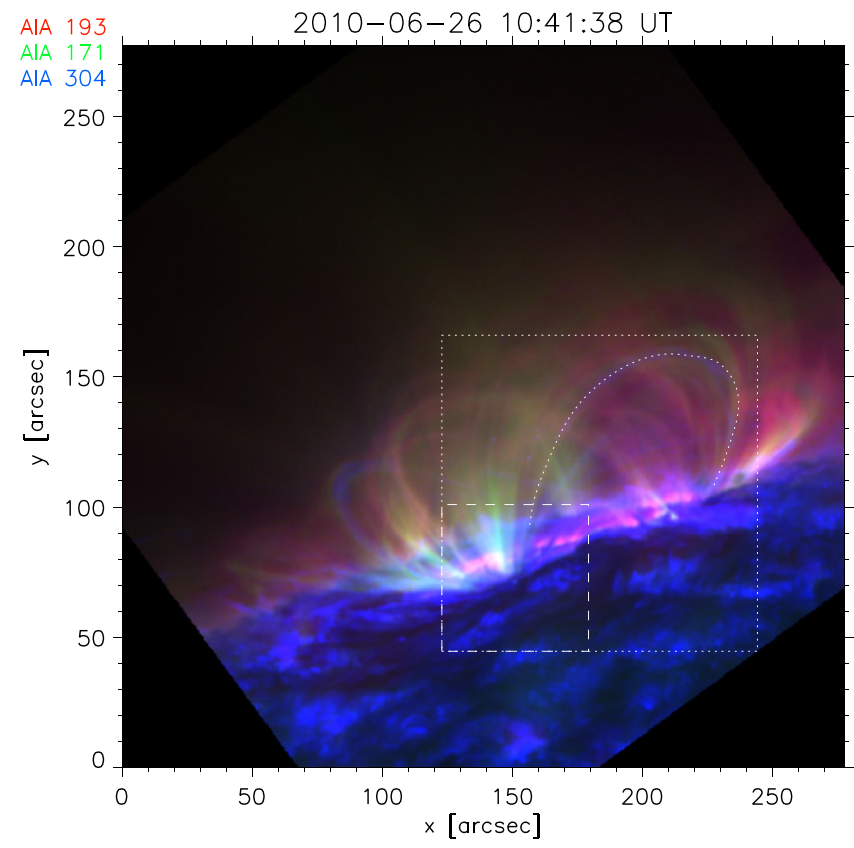

Figure 1. Composite image from SDO/AIA combining three AIA filters, 193 (red), 171 (green), and 304 (blue), showing data set 1: AR 11903 at the east limb on 2010 June 26. The dotted square denotes the field of view of Figure 3. The dotted loop corresponds to loop 2 studied in Section 3.2. The dashed square denotes the field of view of SST.

(An animation of this figure is available.)

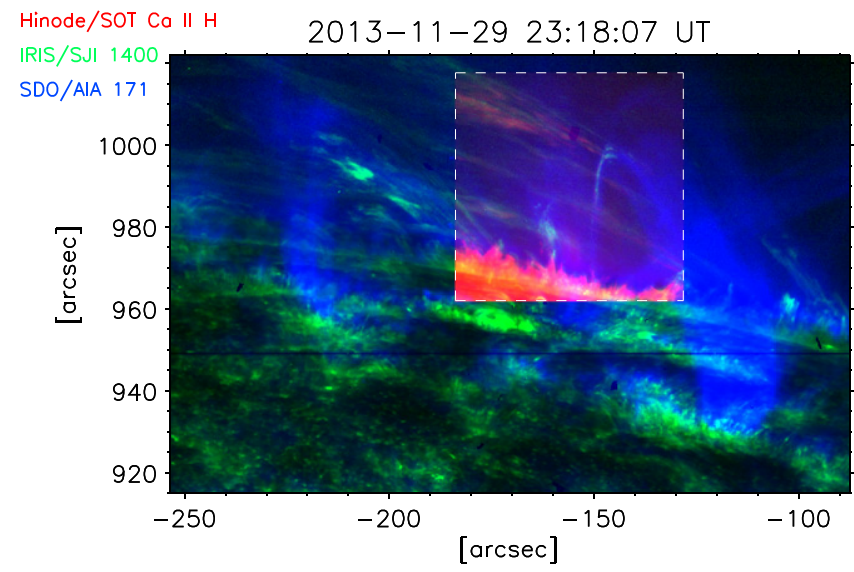

Figure 2. Composite image combining AIA 171 (blue), IRIS/SJI 1400 (green), and Hinode/SOT Ca II H (red), showing data set 2: AR 11903 at the West limb on the 2013 November 29 . The dashed square denotes the field of view covered by Hinode/SOT. The loop with coronal rain toward the center right of the square corresponds to the loop studied in this data set.

(An animation of this figure is available.)

usually spanned by thermally unstable plasmas. The upper photospheric filters 1600 and 1700 were also used for coalignment purposes (see Section 2.2). Level 1.5 data from the SDO/AIA was used, obtained through the normal calibration routines in SolarSoft. Full disk images are provided from AIA with a cadence of $12 \mathrm{~s}$, an image scale pixel of $0 . \prime 6$ pixel $^{-1}$, and a spatial resolution of 1 .' $3-1$ ". 7 .

IRIS combines an SJI and a spectrograph ( $\mathrm{SG}$ ). The IRIS data used here comprises only the SJI instrument since the IRIS slit was not located on the region of interest. Therefore, in this work only the coronal rain imaging capabilities of IRIS are shown. The data set corresponds to level 2 data from a sit-and- stare observation combining the SJI 1400, 1330, and 2796 filters, dominated respectively by the Si IV TR lines 1393.78 and $1402.77 \AA$ (formed around $\log T=4.8$ ), the $\mathrm{C}_{\text {II }}$ chromospheric lines 1334.53 and $1335.71 \AA$ (formed around $\log T=4.3$ ), and the $\mathrm{Mg}$ II $\mathrm{k}$ chromospheric line $2796.35 \AA$ (formed around $\log T=4$ ). IRIS/SJI provides an FOV of $175^{\prime \prime} \times 175^{\prime \prime}$, an image scale pixel of 0 ". 166 pixel $^{-1}$ and a spatial resolution of 0 ". 33 (for the FUV) -0 ". 4 (for the NUV). The cadence for this observation is $36.5 \mathrm{~s}$ in each filter.

CRISP (Scharmer et al. 2008) at SST sampled the $\mathrm{H} \alpha$ spectral line at 41 line positions with $0.085 \AA$ steps $\left(3.9 \mathrm{~km} \mathrm{~s}^{-1}\right)$, in the spectral window $[-1.7,1.7] \AA(\simeq \pm 78$ $\mathrm{km} \mathrm{s}^{-1}$ ) with a cadence of $9 \mathrm{~s}$. The FOV of SST for the present observation is $53^{\prime \prime} \times 57^{\prime \prime}$. The images benefited from the SST adaptive optics system (Scharmer et al. 2003b) and the image restoration technique Multi-Object Multi-Frame Blind Deconvolution (van Noort et al. 2005). Although the observations suffered from seeing effects, most of the images are close to the theoretical diffraction limit for SST at the wavelength of $\mathrm{H} \alpha$ : $\lambda / D \simeq 0$." 14 . We employed the same reduction procedure as in Paper I, which used early versions of parts of the data pipeline CRISPRED (de la Cruz Rodríguez et al. 2015).

Hinode/SOT recorded filtergrams in the $\mathrm{Ca}$ II $\mathrm{H}$ band (formed around $\log T=4$ ) at a cadence of $4.8 \mathrm{~s}$. The image scale pixel is 0 ". 109 pixel $^{-1}$ and the spatial resolution is $\lambda / D \simeq 0$." 2 . The SOT FOV for this observation is 55 " $8 \times 55$." 8 . Processing of data was carried out through the normal calibration routines in SolarSoft.

\subsection{Co-alignment Procedures}

The co-alignment of SST/CRISP and SDO/AIA data for the 2010 June 26 first consisted of determining the solar- $x / y$ coordinates and locating the solar north direction for each CRISP frame. We then eliminated the SST drift by fixing each frame on the sunspot of AR 11084, at the center of the SST FOV, to reduce the frame-to-frame motion of the loop's footpoints (and therefore of the loops themselves). The location of the solar limb for each frame was also determined, which allowed us to apply a gradient filter, reducing the intensity of on-disk features for better visualization of the offlimb structures. SDO data was then cropped and interpolated to the exact SST FOV. Small misalignments still remain, which are then corrected through cross-correlation using photospheric bright points (with long enough lifetime) and the solar limb location (using the AIA filters 1600 and 1700, in combination with the far wing positions of $\mathrm{H} \alpha$, which mostly show the photosphere).

For the SOT-SJI-AIA co-alignment, we first removed the slow drift of SOT by co-alignment of the images through tracking the movement of the limb. The cumulative offsets from this drift were applied to the time series, resulting in a rigid co-alignment on the order of one pixel or less. The IRIS/ SJI images do not have this slow drift, but occasional wobble shifted the images a few pixels (this is normal during eclipse season). Such shifts were measured by applying crosscorrelation techniques and applied to the time series. Then, to co-align the images from different instruments the following procedure was used. First, the AIA images were co-aligned and cropped to the SJI FOV by identifying common bright points in the AIA 1600 filter and the SJI 1400 filter. Afterward, both AIA and SJI images were interpolated to SOT's image pixel 
scale and were co-aligned using common features on the SJI 1400 filter and SOT's Ca II H filter.

\subsection{Methods}

Due to the complex interplay of forces in loops coronal rain dynamics can be extremely complex, especially when observing at sub-arcsecond resolution. Furthermore, their thermodynamic state is also expected to change, as we show in this paper, and as has been also indicated in Harra et al. (2014). A consequence of this is that it is very difficult to follow a single rain clump (at high resolution) over long distances. The clumps will usually split, merge, and elongate along their paths. For these reasons, especially for the CRISP data where myriads of small clumps are observed and for which the line-of-sight (LOS) superposition is significant, individual tracking is very difficult and time consuming. For automatically detecting rain in the CRISP data we have therefore opted for perpendicular cuts across the loops at positions where rain is observed, rather than longitudinal paths along individual clumps. Detection is done through semi-automated procedures verifying specific conditions for the clumps. These consist especially of contrast thresholds (a rain pixel needs to be significantly brighter or darker with respect to the background, depending on whether the detection is realized off-limb or on-disk, respectively; all frames with bad seeing are then removed), intensity variation thresholds (a rain pixel must present an average change in intensity above a specific threshold within a reasonably small time interval), and size restrictions (a clump must span at least three rain pixels in the direction perpendicular to the direction of propagation). Once the rain pixels are identified the clump sizes are calculated with Gaussian fits along and across the direction of propagation. Therefore, one size value for a clump is the average over several tens or hundreds of measurements. The results are also tested for specific conditions, such as size thresholds, small fit errors, consistent widths (a clump's width must not differ significantly over a short distance along the axis of propagation), and so on. For rain detection with CRISP we have further opted for using Doppler intensities rather than the clump intensities alone, since detection is improved in this way. The Doppler intensity for a rain pixel at position $x, y$ and time $t$ is defined as $I_{\text {Dopp }}(x, y, \lambda, t)=I(x, y, \lambda, t)-I\left(x, y, \lambda_{0}-\left(\lambda-\lambda_{0}\right), t\right)$, where $\lambda_{0}$ is the theoretical wavelength value at rest.

For the rain observed with SOT, SJI, and AIA we have opted for the "traditional" method used in previous studies. First, individual clumps' paths are traced manually. For each time step at which rain appears along the path and at each position along the clumps' paths semi-automated routines measure the width and length following the same criteria specified above. The average and standard deviation of these measurements for a given path provide the clump's size (width and length) and corresponding errors.

For all the above measurements, regardless of the instrument, clump intensities are calculated by subtracting from a given rain pixel intensity the average background intensity at the same pixel position. The average is calculated over a time interval close to that of detection and for which no rain is detected. The Doppler velocity and temperature calculation with CRISP were performed following the same technique as in Antolin et al. (2012) and we refer the reader to that paper for details. It is important to note that with this technique upper limits to the temperatures are provided, since the microvelocities from turbulence are ignored.

For the follow-up of individual rain features we used the CRisp SPectral EXplorer (Vissers \& Rouppe van der Voort 2012) and its auxiliary program TANAT (Timeslice ANAlysis Tool). Both are widget-based tools, which enable the easy browsing and analysis of the image and spectral data, the determination of clump paths, extraction, and further analysis of spacetime diagrams. Fitting piece-wise segments along the clumps trajectories in the spacetime diagrams we can estimate the projected velocities (in the plane of the sky). With this method, the error (standard deviation) for projected velocities is estimated to be roughly $5 \mathrm{~km} \mathrm{~s}^{-1}$.

\section{TEMPERATURE EVOLUTION}

\subsection{SDO/AIA and SST/CRISP Observations}

In Figure 1 and Animation 1 active region AR 11903 is viewed at the east limb. The figure is a composite of three AIA filter images, combining the 193 filter (in red), dominated by the coronal Fe XII 193.509 $\AA$ emission at $\log T=6.2$, the 171 filter (in green), dominated by the Fe IX $171.073 \AA$ emission at $\log T=5.9$, and the 304 filter (in blue), dominated by the TR He II $304 \AA$ emission at $\log T=4.9$. The animation clearly shows that a significant quantity of the coronal volume in the active region, filled by loops, exhibits continuous intensity variation. Such dynamical state is strongly suggestive of loops out of hydrostatic equilibrium (Aschwanden et al. 2001), and possibly in a thermal non-equilibrium state. This is further supported by the fact that large parts of the coronal volume change from red to green and to blue, indicative of cooling.

During this observation, SST was pointing at the footpoint region of these loops, as indicated in Figure 1, where a sunspot is located. The sunspot is roughly located in the middle of the FOV.

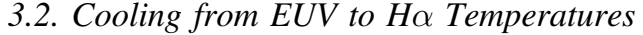

To investigate the thermal non-equilibrium scenario we have chosen a loop (hereafter loop 2, which occurs after loop 1, defined later on) in the large $S D O$ FOV, which exhibits this gradual change of intensity from the hot to the cool AIA channels. This loop is highlighted in Figure 1 in dotted lines, and it extends $\approx 42 \mathrm{Mm}$ above the surface leading to an approximate length of $132 \mathrm{Mm}$ for a circular geometry. Its left footpoint is also covered by the FOV of SST. A zoom-in figure focusing on the loop is shown in Figure 3, where two snapshots in the loop evolution can be seen in the upper two panels, separated by $\approx 24 \mathrm{~min}$, while the lower panel shows the integrated intensity in different AIA channels along the top part of the loop, as well as the size-weighted coronal rain intensity in $\mathrm{H} \alpha$ (defined later on) close to the loop footpoint. These panels clearly show a progression in the AIA channels from 193 dominated emission to 304 dominated emission. Prior to $t \simeq 15$ min the loop top is only visible in 193 but decreasingly so between $t=10-17$ min. From that point on, 171 starts dominating clearly until peaking at $t=25.8 \mathrm{~min}$. Only when 171 has already decreased to a local minimum plateau do we observe an increase in 304 intensity at the top, which takes over from $t=41.0 \mathrm{~min}$ and peaks at $t=50.6 \mathrm{~min}$, close to $25 \mathrm{~min}$ after the peak in 171 signal. The first sign of coronal rain in $\mathrm{H} \alpha$ in this loop is already detected prior to the 304 peak. 

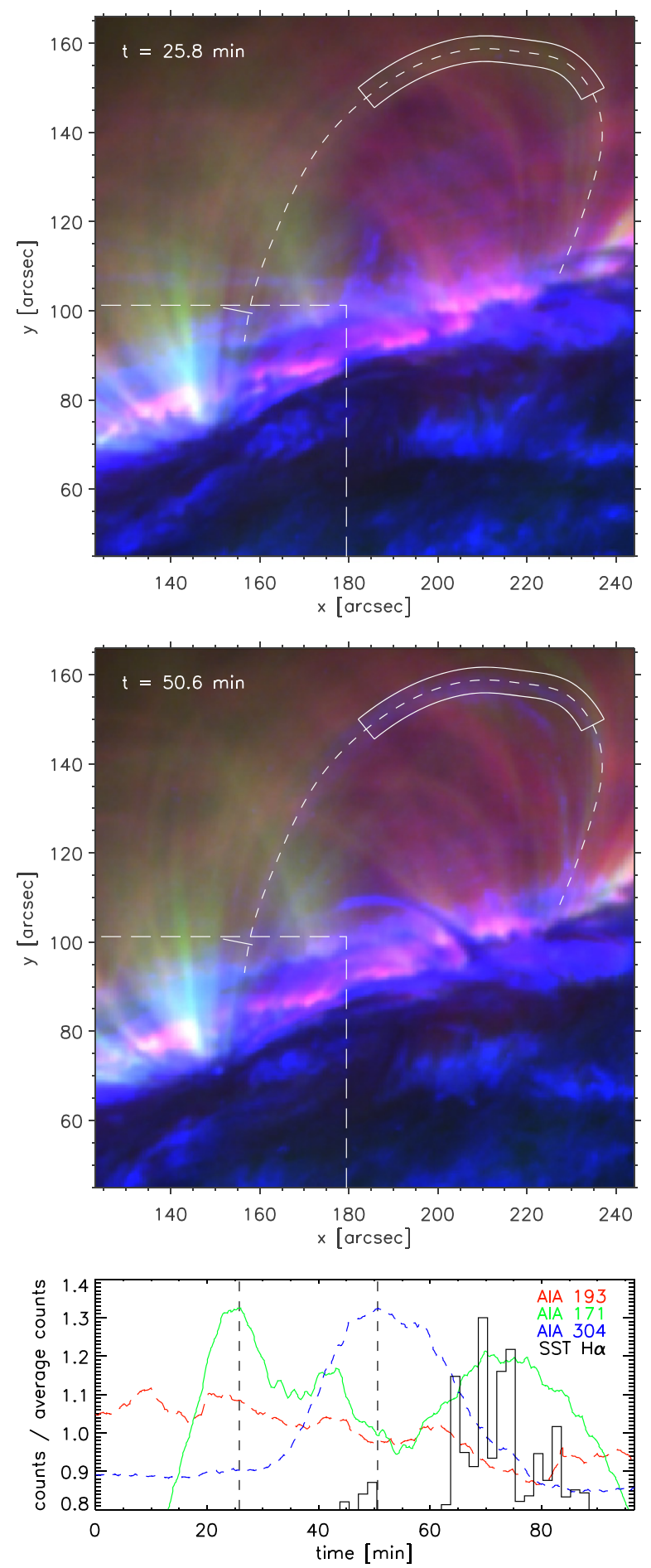

Figure 3. Evolution of a loop hosting coronal rain in three AIA diagnostics and CRISP H $\alpha$ for data set 1. The top two panels show the loop (defined as loop 2 in the text) at two instances in time (indicated by vertical dashed lines in the lower panel) in AIA 193 (red), 171 (green), and 304 (blue), with the loop path traced by the curved dashed line in the upper two panels. The long-dashed box outlines the extent of the SST field of view, the lower left corner of which coincides with that of the panels. The lower panel shows the counts normalized to their average as function of time for AIA 193 (long-dashed red), 171 (solid green) and 304 (dashed blue), where these intensities have been obtained over the box around the loop top in the upper panels. For CRISP $\mathrm{H} \alpha$ (solid black) we plot a histogram of intensities (scaled by 0.5 and shifted by +0.8 to fit nicely in the panel) that stem from measurements across the short white line perpendicular to the loop path located at about $\left(155^{\prime \prime}, 100^{\prime \prime}\right)$ (which corresponds to cut C2 in Figure 5), and are weighted by the respective clumps' widths squared (see Figure 7 for further details).
While AIA 304 has a single-peaked light curve, AIA 193 and 171 show much more variability on the scale of a few to 10-15 min. The variations in $\mathrm{H} \alpha$ are much more extreme and on a shorter timescale than for the AIA channels, but this is to be expected considering the difference in cadence and the fact that the AIA intensities are integrated over a large area, while the $\mathrm{H} \alpha$ intensities stem from single rain clump measurements. A large quantity of $\mathrm{H} \alpha$ clumps is observed falling across the SST FOV with basically two slightly different trajectories but converging to similar locations, apparently within the umbra of the sunspot. In Figure 4 an example of a large clump is shown in an $\mathrm{H} \alpha$ Doppler image $( \pm 0.76 \AA$, left panel $)$ and in AIA filters. The track of the clump, shown in dotted lines coincides with a dark loop structure of larger width in the EUV passbands. This dark structure matches with a bright loop structure above the limb (hereafter loop 1), adjacent (to the right) to loop 2. A composite image of the SST/CRISP FOV (Doppler image $\mathrm{H} \alpha \pm 0.76 \AA$ in red) together with AIA 171 (in green) and AIA 304 (in blue) is shown in Figure 5, where the same rain event as in Figure 4 is shown.

In order to identify all the clumps, for instance those belonging to loops 1 and 2, a semi-automatic routine was written detecting all rain-like structures crossing a specific path in the FOV (see Section 2.3). This routine calculates the intensity, Doppler velocity, the width, and the length for each clump. We select two cuts, $\mathrm{C} 1$ and $\mathrm{C} 2$, roughly transverse to loops 1 and 2, respectively, plotted in Figure 5, crossed by most of the clumps. Animations 3 and 4 show the detected clumps as they cross $\mathrm{C} 1$ and $\mathrm{C} 2$, respectively (in red the FWHM of the fit to the clumps' widths). These cuts can be seen to be roughly perpendicular to the trajectory of the clumps. The algorithm confidently detects 46 and 95 clumps for $\mathrm{C} 1$ and $\mathrm{C} 2$, respectively (as shown in the animation, a myriad of smaller clumps exist, but these are too faint and cannot be confidently detected by the algorithm). Despite the apparent crossing of these loops (evidenced by the intersection of $\mathrm{C} 1$ and $\mathrm{C} 2$ ), these are actually well separated along the LOS (the algorithm further ensures that no clumps are common to both $\mathrm{C} 1$ and $\mathrm{C} 2$ ). This is evidenced by the different Doppler velocities between clumps belonging to separate loops. Indeed, clumps belonging to a specific loop will share a common trajectory and therefore a similar Doppler velocity in general. This correlation is also obtained in three-dimensional (3D) numerical simulations of thermal instability reproducing coronal rain and prominence threads (Luna et al. 2012). Figure 6 shows a histogram of Doppler velocities and derived temperatures for all detected clumps. Positive and negative values correspond, respectively, to redshifts and blueshifts. We can see that the Doppler velocities concentrate in three different groups, one peaking at $\approx 30 \mathrm{~km} \mathrm{~s}^{-1}$, another at $\approx-10 \mathrm{~km} \mathrm{~s}^{-1}$, and the last one at $\approx-40$ $\mathrm{km} \mathrm{s}^{-1}$. The first and second groups correspond to the clumps crossing $\mathrm{C} 1$ and $\mathrm{C} 2$ respectively, while the third corresponds to the Doppler velocities of the clumps in loop 2 at chromospheric heights (cut F2, defined in Section 4.4). The projected velocities of the clumps crossing $\mathrm{C} 1$ and $\mathrm{C} 2$ are found to be similar, with an average of $110 \mathrm{~km} \mathrm{~s}^{-1}$. This ensures that the angles of fall for each family of clumps is different, and similar within each family (as indicated by the formulae for the falling angle calculated in Paper I). We conclude therefore that the $\mathrm{C} 1$ and $\mathrm{C} 2$ clumps correspond to two different loops.

The clumps crossing $\mathrm{C} 2$ are found to follow a dark structure in the EUV passbands similar to that plotted in Figure 4. This 


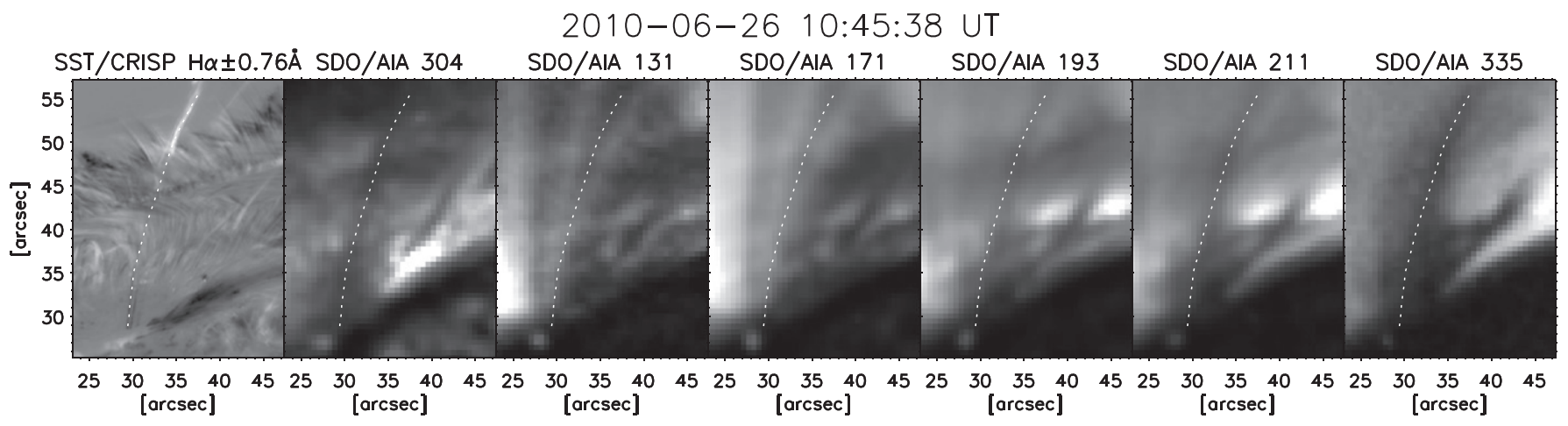

Figure 4. Multi-panel figure showing a snapshot of a clump falling toward the sunspot in data set 1. From left to right, a Doppler image from SST/CRISP in H $\alpha \pm 0.76 \AA$, SDO/AIA 304, 171, 193, 211, and 335. The dotted lines follow the center of the condensation, observed in emission above the spicular layer (above $\left.y \approx 47^{\prime \prime}\right)$ and in absorption below this layer. Notice the kink in the trajectory of the clump at $y \approx 35^{\prime \prime}$.

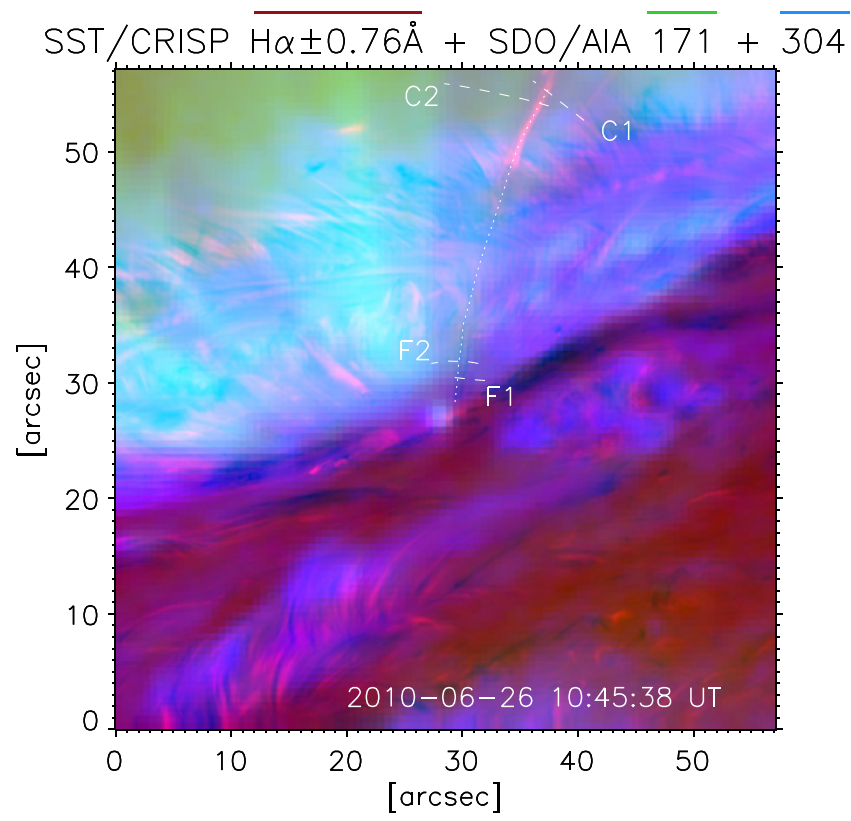

Figure 5. Composite image combining a Doppler image from SST/CRISP in $\mathrm{H}$ $\alpha \pm 0.76 \AA$ (in red), SDO/AIA 171 (in green) and SDO/AIA 304 (in blue) for data set 1 . The image shows the same snapshot of the falling clump as in Figure 4, whose trajectory is indicated by the dotted curve. Four transverse cuts to the main axis of two rainy loops are shown, located at two different heights: $\mathrm{C} 1$ and $\mathrm{F} 1$ for loop 1 ( $\mathrm{C} 2$ and F2 for loop 2) at coronal and chromospheric heights, respectively. The observed clump belongs to loop 1. Animations 3-6 are zooms into this figure, showing the detected clumps crossing cuts $\mathrm{C} 1, \mathrm{C} 2$, F1, and F2, respectively (where the FWHM of the fit to the clumps' widths is plotted over in red).

(Animations a, b, c, and d of this figure are available.)

dark EUV structure appears bright above the limb and matches with loop 2. The geometry of the loop seen in Figure 3 and the direction of the flow suggest that the material is coming toward the observer, matching with the negative Doppler velocities found in Figure 6 for the $\mathrm{C} 2$ clumps. We can therefore confirm that the $\mathrm{C} 2$ clumps belong to loop 2.

As shown by Anzer \& Heinzel (2005) the $\mathrm{H} \alpha$ intensity from filaments is strongly correlated to darkening in EUV passbands, and is due mainly to continuum absorption from neutral hydrogen, neutral helium, and singly ionized helium (and to a lesser extent to volume blocking, especially in the case of small size structures as coronal rain clumps). Therefore, both the volume and the composition of the emitting structure are important factors that can be correlated to the detected intensity variations in the EUV passbands. In the lower panel of Figure 3 we thus plot as a black histogram the $\mathrm{H} \alpha$ intensity multiplied by the width squared (assuming axi-symmetry for the clump) for the clumps crossing $\mathrm{C} 2$. The bulk of the distribution spans over $25 \mathrm{~min}$ and the peak is shifted by $\approx 20 \mathrm{~min}$ from the 304 peak. However, the $\mathrm{H} \alpha$ detection is made at the footpoints, while the AIA peaks are first observed at the apex. The clumps are observed to fall with average total speeds of $110 \mathrm{~km} \mathrm{~s}^{-} 1$ and accelerations around $0.1 \mathrm{~km} \mathrm{~s}^{-2}$. Assuming no initial velocity, constant acceleration and a circular loop, the travel time of the cooling plasma is $20 \pm 10 \mathrm{~min}$, which locates in time the origin of the clumps close to the 304 peak. This result supports the common origin for the $\mathrm{H} \alpha$ clumps with the rain observed in 304 in loop 2.

The temperature histogram in Figure 6 shows that all the detected clumps have very cool temperatures, mostly below $10,000 \mathrm{~K}$, with a peak at $2000-5000 \mathrm{~K}$. As explained in Section 2.3 these temperatures correspond to upper limits. While we postpone a discussion on these low-temperature values to the pertinent section, these results clearly indicate that loops 1 and 2 undergo full catastrophic cooling to chromospheric temperatures.

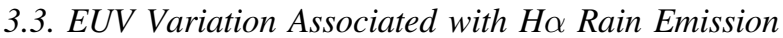

It is interesting to compare the EUV and $\mathrm{H} \alpha$ intensity variations across the $\mathrm{C} 1$ and $\mathrm{C} 2$ segments. This is shown in Figure 7. For each time step we average the intensity in each channel over a small region centered around each cut. For $\mathrm{H} \alpha$, as in Figure 3, we plot the average of the intensity times the square of the width for each clump (see figure caption for more details). Also, for the times at which a clump is detected the intensity in each EUV channel is calculated over the location of the clump only, in order to discern better a possible effect on the EUV passband for the passing clump. For the clumps crossing $\mathrm{C} 1$ we can see that most of the time groups of clumps close in time produce a decrease in all of the EUV intensity channels (at times $t \approx 0,10,27,40 \mathrm{~min}$ ), except 304, for which an intensity increase is generally detected. For $\mathrm{C} 2$ a clear correlation is also detected. Quasi-periodic fluctuations in the EUV passbands observed in the time range $t=60-90 \mathrm{~min}$ seem to match the $\mathrm{H} \alpha$ intensity fluctuation in the same time range. Contrary to the previous case here a maximum in the $\mathrm{H} \alpha$ intensity seems to be positively correlated with all EUV 

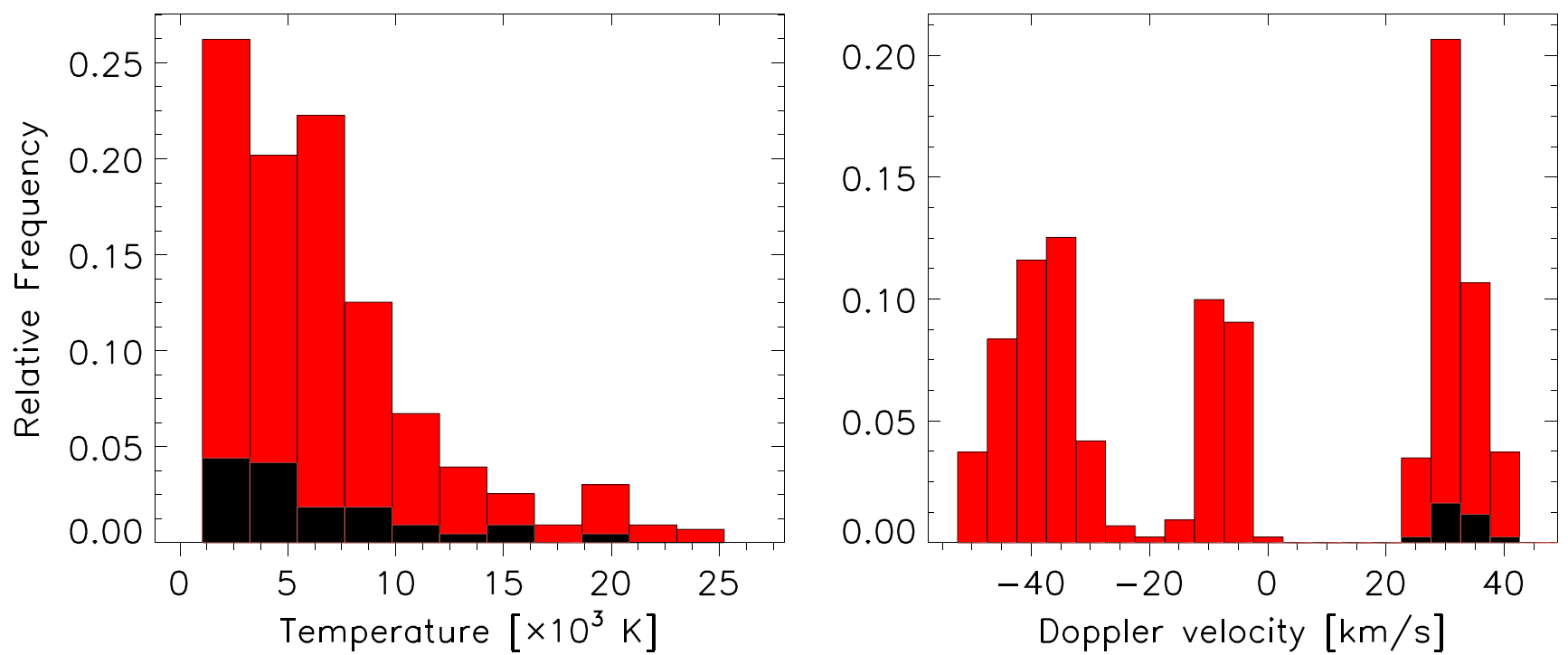

Figure 6. Histograms of temperature (left) and Doppler velocity (right) for the $\mathrm{H} \alpha$ clumps detected with SST/CRISP in data set 1, following the method described in Section 2.3. The black histogram corresponds to events whose measurements have a standard deviation above $10 \%$ of the mean. Positive and negative Doppler velocities correspond, respectively, to redshifts and blueshifts.

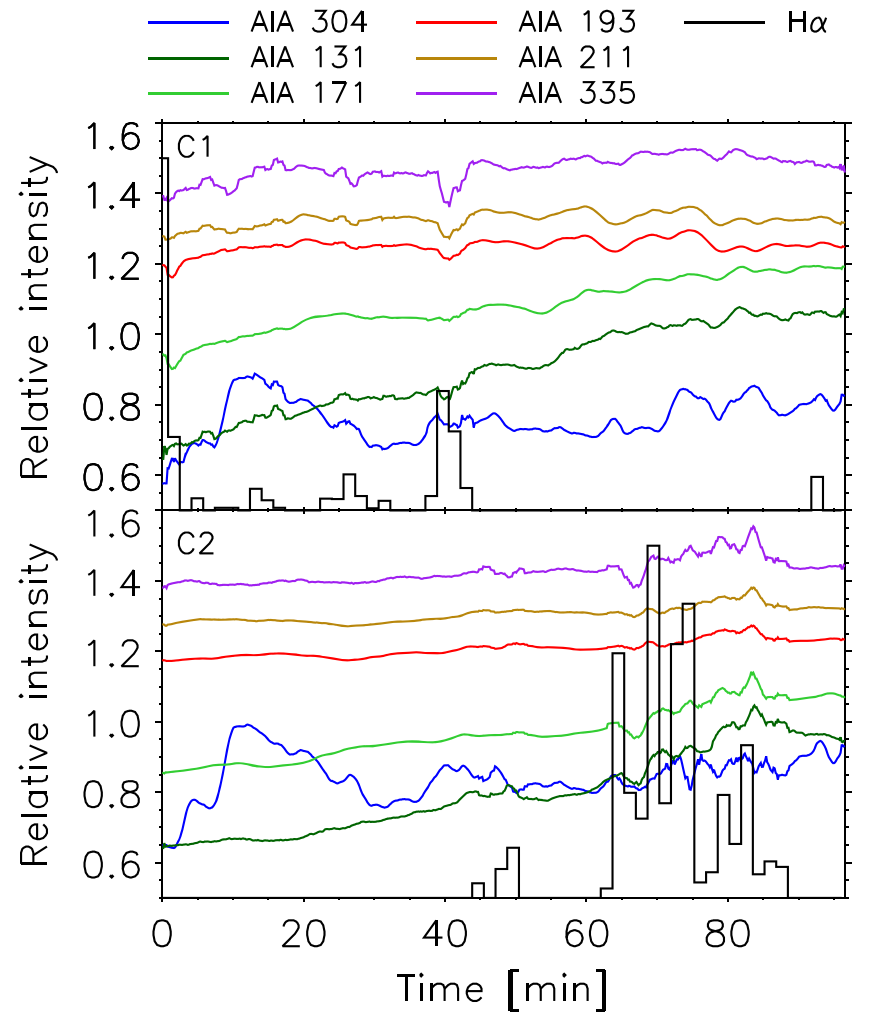

Figure 7. Time evolution of the intensity across cuts $\mathrm{C} 1$ and $\mathrm{C} 2$ (corresponding to loops 1 and 2 in data set 1, respectively) for multiple AIA filters (indicated at the top with their respective color) and the condensations detected with CRISP in $\mathrm{H} \alpha$ (in black). The histogram of $\mathrm{H} \alpha$ intensities is constructed by taking a bin size of a $100 \mathrm{~s}$, and for each time bin the intensity of all contained clumps are summed, each multiplied by the clump's width squared. The AIA intensities at each time step are averages over a region of $1 . " 8$ in width in the transverse direction to the cuts and same length as the cut, except at times where condensations are detected in $\mathrm{H} \alpha$. For these, the average is not calculated over the entire cut but limited to the positions along the cut where the clump is detected (defined by the width and the location of the clump). passbands. Indeed, each peak of the former seems to correspond to a local peak in the latter.

\subsection{Hinode/SOT, IRIS/SJI, and SDO/AIA Observations}

We now turn to the second set of observations involving SOT, SJI, and AIA. A look at Figure 2 and Animation 2 immediately indicates the large presence of cool material above the active region. In red, green, and blue we show $\mathrm{Ca}$ II $\mathrm{H}$ emission from SOT, Si Iv 1400 emission from IRIS/SJI, and Fe Ix 171 emission from AIA, respectively. We can see the presence of two kinds of cool structures above the spicular layer. Prominence material is observed flowing relatively slowly roughly horizontally, while coronal rain is observed falling relatively faster along loops bright in AIA 171 (other cool static prominence-like structures are also observed, e.g., at $(x, y) \approx(-160,970))$. Here we will concentrate on a loop exhibiting coronal rain, with one footpoint at $(x, y) \approx$ $(-120,930)$ and the other apparently at $(x, y) \approx(-150,955)$, expanding in the corona up to a height of roughly $40^{\prime \prime}$ (leading to a length of roughly $180 \mathrm{Mm}$ assuming circular geometry). The rain appears from 23:12 UT to 23:30 UT (the end of the co-observation sequence) and is observed to initiate at the apex of the loop and to fall toward the farther footpoint.

A zoom-in onto the loop of interest is shown in various wavelengths in Figure 8 and Animation 7. Contrary to the first data set, here we are able to observe the chromospheric emission from the rain well above the spicular layer, but we are not able to follow it once it goes below this layer.

\subsection{Cooling Through Transition Region Temperatures}

In order to follow closely in time the evolution of temperature in each wavelength we plot in the upper panel of Figure 9 the integrated emission for each filter for coronal rain pixels belonging to the previously indicated loop. As seen in Animation 2, halfway through the coronal rain event a 


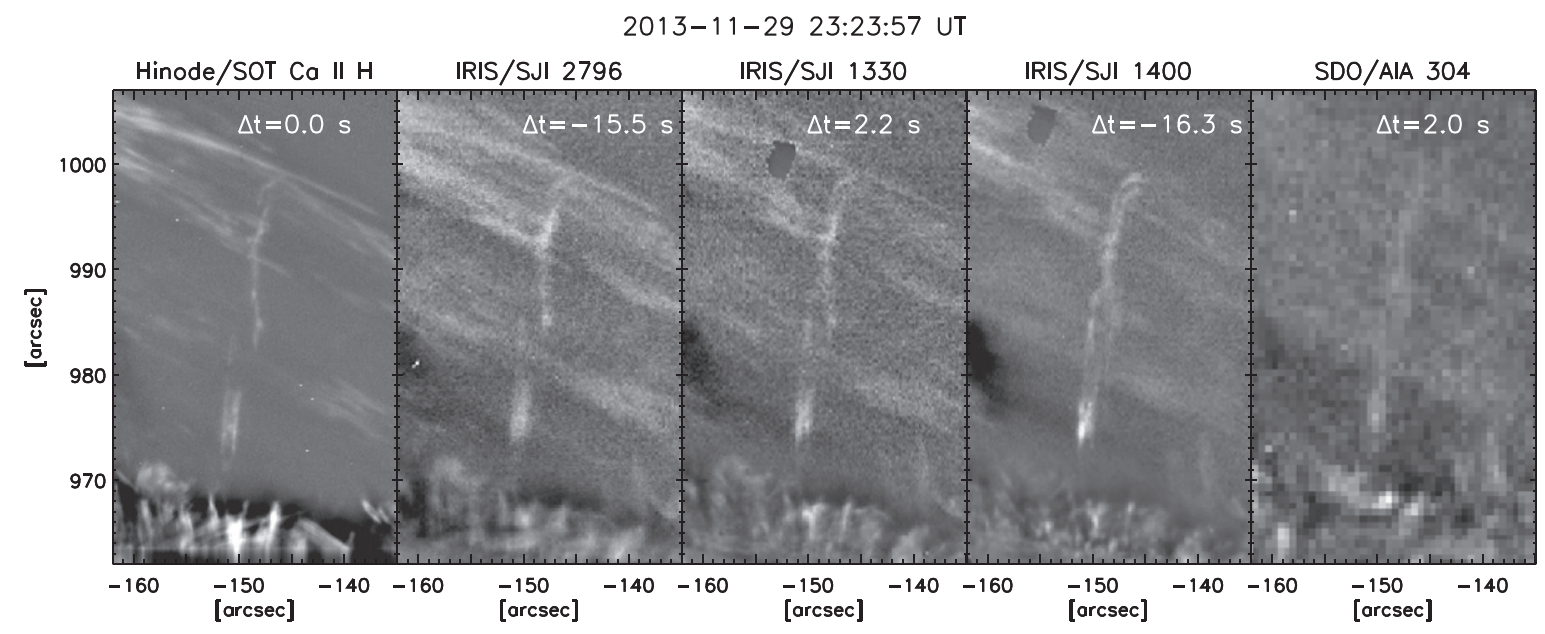

Figure 8. Multi-panel showing a snapshot of the coronal rain event in data set 2. From left to right we have Hinode/SOT in Ca II H, IRIS/SJI 2796, 1330, 1400, and SDO/AIA 304. Each snapshot corresponds to the closest snapshot in time to that taken by SOT, and for each the average background is subtracted. The time difference of each snapshot with respect to that of SOT is indicated in the upper part of each panel.

(An animation of this figure is available.)

prominence that appears to be in the background crosses the upper portion of the loop. In order to minimally reduce such LOS projection effects, the selection of clump paths and the times and locations of rain along such paths is done manually and is done ensuring that the prominence is not along the LOS. In the figure we can see that the first emission of rain appears in the IRIS/SJI images, before showing up in the $\mathrm{Ca}$ II $\mathrm{H}$ filter. The SJI 1400 filter comes first (at $t_{0} \approx 44.5 \mathrm{~min}$ ), followed by a peak in SJI $1330\left(t=t_{0}+2.5 \mathrm{~min}\right)$, then SJI 2796 $\left(t=t_{0}+5.5 \mathrm{~min}\right)$ and a small peak in Ca II $\mathrm{H}$ roughly at the same time. The light curves generally show short-timescale variability. While all the SJI channels have a first large peak followed by gradually smaller peaks, the $\mathrm{Ca}$ II $\mathrm{H}$ emission starts with a small peak and increases over the next five minutes, with the highest peak at $t=t_{0}+8 \mathrm{~min}$. Interestingly, the time lag between the first or highest peak of each diagnostic seems to increase as the rain becomes visible in each: the $\mathrm{C}_{\text {II }}$ peak follows the $\mathrm{Si}$ Iv peak after $\sim 2.5 \mathrm{~min}, \mathrm{Mg}$ II $\mathrm{k}$ follows $\mathrm{C}$ II after $\sim 3 \mathrm{~min}, \mathrm{Ca}$ II $\mathrm{H}$ follows $\mathrm{Mg}$ II $\mathrm{k}$ after $\sim 3-3.5 \mathrm{~min}$. Although the dynamic range differs, part of the increases and decreases are visible accross several diagnostics. For instance, the initial $\mathrm{C}_{\text {II }}$ peak around $46.5 \mathrm{~min}$ seems to coincide roughly with a secondary $\mathrm{Si}$ IV peak, the initial $\mathrm{Mg}_{\text {II }} \mathrm{k}$ increase and peak around $49 \mathrm{~min}$ is mirrored in minor by $\mathrm{Ca}$ II $\mathrm{H}$, while AIA 304 and 171 seem to follow each other closely throughout.

The behavior of the AIA filters, and especially 171, is strikingly different. Animation 2 shows that most of the loop is bright and stays bright in 171 , from a first peak about $10 \mathrm{~min}$ before first appearance of coronal rain in SJI 1400. No EUV darkening co-spatial to the rain is observed in this case, as was the case for data set 1 . Both AIA 171 and 304 increase slightly in intensity during the rain event, with the 304 emission of the rain starting at $t=t_{0}+3.5 \mathrm{~min}$, roughly at the same time as the Mg II $\mathrm{k}$ emission. This is more clearly seen in Animation 7. This behavior seems contrary to that observed in the loops in the first data set with CRISP and AIA, in which 171 emission at the loop apex peaks either prior or following a rain event and is at a minimum during the rain event, and in which 304 peaks after 171 and roughly at the same time or prior to $\mathrm{H} \alpha$ (cf. Figure 3). Multiple small peaks are also observed in the AIA 171 emission, in a quasi-periodic fashion separated by a few
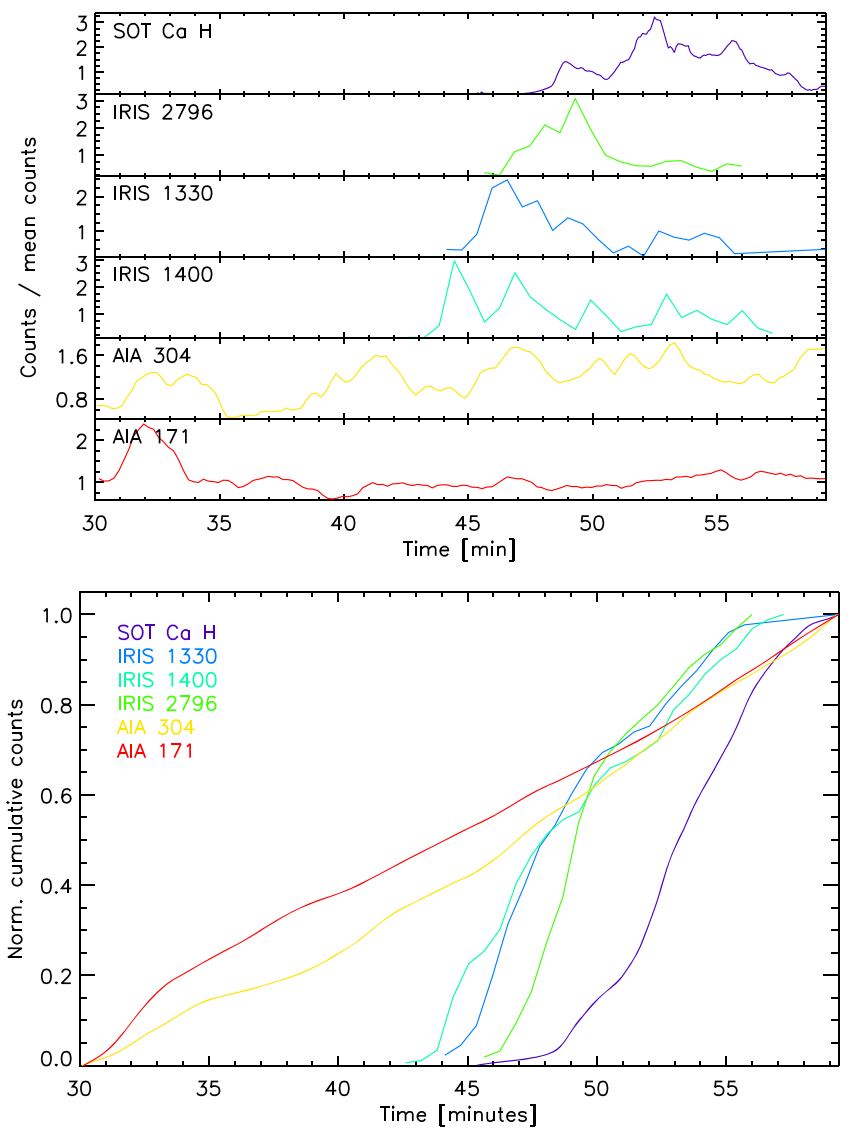

Figure 9. Light curves (top panel) and cumulative light curves (bottom panel) of the coronal rain pixels in the loop highlighted by the rain in Figure 8 of data set 2. In both panels the Hinode, IRIS, and SDO diagnostics are differentiated by color: $\mathrm{Ca}$ II $\mathrm{H}$ (purple), C II 1330 slit-jaw (blue), Si Iv 1400 slit-jaw (cyan), Mg II k 2796 slit-jaw (green), AIA 304 (yellow), and AIA 171 (red). The SOT and AIA light curves have been smoothed over seven and three time steps, respectively, close to the cadence of the IRIS slit-jaw images.

minutes, similar to the behavior observed in data set 1 , shown in Figure 7.

Another useful way to visualize the evolution of emission is through the cumulative light curves, plotted in the lower panel 
of Figure 9. In this figure we can see the same progression throughout the filters, with the variability reflected now in the slope of each light curve. Accordingly, the behavior between the SJI filters is very similar, characterized by two different slopes: an initial fast rise with a steep slope (until $t \approx 50 \mathrm{~min}$ ) followed by a more gradual rise. The fast rise can also be seen in Animations 2 and 7: the rain brightens as soon as it appears close to the apex of the loop, especially in SJI 1400 and 1330. This two-step behavior suggests two different cooling phases. The $\mathrm{Ca}$ II $\mathrm{H}$ emission appears mostly during the second cooling phase, it has roughly the same slope throughout the evolution, reflecting a similar rise and decay of the intensity in the upper panel. Accordingly, Animations 2 and 7 show a more gradual emission in $\mathrm{Ca}$ II $\mathrm{H}$. The behavior between the AIA 304 and 171 filters is very similar and constant on average, leading to a roughly constant slope in the cumulative plot of Figure 9.

Figures 8, 9 and Animation 7 further show that the emission in all filters co-exists in the loop during most of the catastrophic cooling event, but especially during the second phase of slow cooling. This strongly suggests a multi-temperature structure for the thermally unstable loop. This is further supported by the increase of the AIA 171 intensity along the loop during the rain event. This will be further discussed in Section 5.2.

The apparent mismatch with the AIA 304 channel, this one occurring $3.5 \mathrm{~min}$ after first occurrence in SJI 1400, could be due to the low sensitivity and lack of resolution of AIA with respect to the other instruments. A small clump will not become bright enough above the noise level unless it either increases in intensity or becomes larger. In Animation 7 we can see that AIA 304 emission is barely visible above the noise level, supporting the interpretation.

\section{MORPHOLOGY}

\subsection{Rain Showers with Cool Cores and Associated Continuous EUV Darkenings}

Figure 3 and Animation 1 show that the downward flow along many of the loops in the active region seen in AIA 304 has a continuous character with little clear substructure, especially in the direction of motion. This is also observed in the lower part of the loops shown in Figure 4, where the downflow appears dark in absorption. However, as revealed by Animations 3 and 4, the falling $\mathrm{H} \alpha$ clumps observed with CRISP come in various sizes and intensities along the same paths. Figure 5 shows an example of a clump in $\mathrm{H} \alpha$ in emission above the spicular layer, and extending onto the disk toward the umbral region of the sunspot (marked by a dotted line in the figure). The on-disk part of the elongated rain clump is observed as a thin absorption feature and is adjacent to other elongated rain clumps (also seen in Figure 4). Surrounding the $\mathrm{H} \alpha$ emission, EUV darkening can be observed all along the clump trajectory in the lower part of the loop. This low EUV emission is more persistent, as shown in Figure 7, thus matching the continuous (as opposed to clumpy) character observed in AIA 304 in the larger FOV of Figure 1. However, as shown in Figure 7 and described in Section 3.3, a close look at this low emission reveals small EUV variations, which appear correlated to the $\mathrm{H} \alpha$ intensities. This occurs especially when several $\mathrm{H} \alpha$ clumps appear close in time. Such groups composed of a large number of small $\mathrm{H} \alpha$ clumps were denoted in Paper I as "showers." As predicted in that work here we show that such showers, and also a small minority of large and dense enough $\mathrm{H} \alpha$ clumps, are associated with absorption features in EUV (and corresponding intensity variations within the dark structure). Figure 4 shows that these absorption features are significantly wider than the $\mathrm{H} \alpha$ emission (on the order of a few times the $\mathrm{H} \alpha$ width), suggesting a wide transition from cooler to hotter temperatures. Also, a comparison between Animations 1 and 3 (or 4) indicate that the EUV absorption features are significantly longer than the $\mathrm{H} \alpha$ clumps (except for the few very long clumps that extend beyond the FOV, for which we cannot confirm). These results may suggest at first a scenario in which cool chromospheric cores are surrounded by warmer diffuse shells. However, the large difference in spatial resolution between CRISP and AIA may be the main factor behind this picture.

\subsection{Co-spatiality of Emission and Substructure}

The previous scenario is further supported by data set 2 . As shown by Figure 8 and Animation 7, the downflow in AIA 304 appears mostly structure-less, although with a slightly brighter head (which also appears bright in the other chromospheric and TR filters). On the other hand, substructure can clearly be seen not only in the chromospheric filters (Ca II H and SJI 2796) but also in the TR filters (SJI 1330 and SJI 1400). Close inspection further indicates a very similar rain structure suggesting a high degree of co-spatial emission in both chromospheric and TR filters. This can more clearly be seen in Figure 10, which shows a composite image of $\mathrm{Ca}$ II $\mathrm{H}$ (in red), SJI 1330 (in blue), and SJI 1400 (in green). This result suggests that the lack of structure in AIA 304 may not be due mainly to a difference in temperature but rather to a lack of spatial resolution.

To analyze more closely the degree of co-spatiality we zoom-in into the region and follow a small shower of clumps (the head of the rain, which appears bright in Figure 8). This is shown in Figure 11, where the same shower is shown at three different times along its fall, separated by $\approx 40 \mathrm{~s}$ from each other. The shower's length becomes shorter as it falls (passing from $8^{\prime \prime}$ to $\left.4^{\prime \prime}\right)$. Its width on the other hand remains mostly unchanged. However, the width of the shower (and the amount of substructure) depends strongly on the wavelength. While in AIA 304 the shower appears as a single entity, the presence of substructure is obvious in $\mathrm{Ca}$ II $\mathrm{H}$. The shower can be seen to be composed of two strands, each one presenting inhomogeneities along their lengths. The substructure is far less evident in SJI 2796 , probably due to the significant increase of opacity in this line. However, it resurfaces in SJI 1330 and SJI 1400 with similar shapes, although less clear due to the lower resolution. This picture not only proves a multi-temperature scenario for coronal rain but also proves the existence of substructure in thermally unstable loops in chromospheric to at least TR temperatures. A look at AIA 171 (Animation 2) further shows that coronal temperatures are present throughout the loop, especially in the wake of the rain head.

\subsection{Density for a Thick Clump}

Through the change in AIA emission produced by EUV absorption from hydrogen and helium in the clumps in data set 1 we can estimate their densities following the technique by Landi \& Reale (2013). The application of this technique is based on certain assumptions. Mainly, the plasma needs to be in ionization equilibrium, the produced absorption needs to be homogeneous along the LOS, and the emission in the 


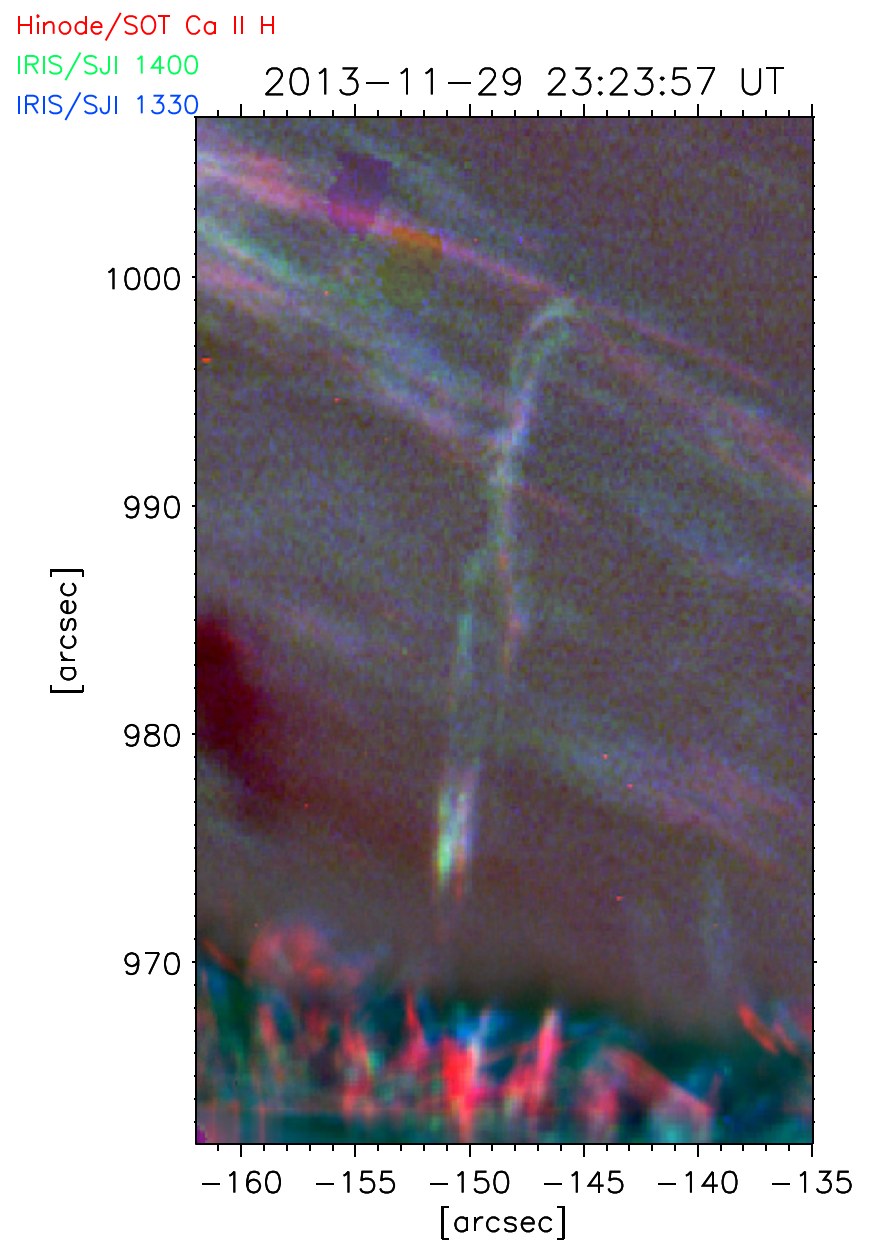

Figure 10. Composite image of the rain event in data set 2 combining Hinode/ SOT in Ca II H (in red), IRIS/SJI 1400 (in green), and IRIS/SJI 1330 (in blue).

(An animation of this figure is available.)

corresponding wavelengths of the absorption needs to be minimal. While these may be questionable in the present case (a catastrophically cooling plasma could be out of ionization equilibrium if the cooling timescale is shorter than the ionization and recombination timescales; also, as shown previously, we have a strong degree of density inhomogeneity within the rain) the results of the applied technique can nonetheless provide important density constraints and clues on the state of the plasma. Here we do this for a large clump producing enhanced EUV darkening, similar to that shown in Figures 4 and 5 , but we postpone a full investigation on densities to future work. In Figure 12 we plot the $L$ function, defined in (Landi \& Reale 2013, formula 9), which, at temperature of absorption is equal to the hydrogen column density. Our case is similar to their special case 2 (Section 3.2 in their paper). The background emission is measured at the same locations where the EUV darkenings are observed but at times where no $\mathrm{H} \alpha$ clumps are observed (we take a few time steps prior and after the passage of the clump, and then take the average of the two). The EUV intensities are taken over the trajectory of the clump, seen as darkened streaks in an $x-t$ plot. In the calculation of $L$ we further assume a helium abundance of $5 \%$. In Figure 12 we can see that the region of intersection of all curves is concentrated in $\log T_{\mathrm{abs}}=4.4-4.6$ and $\log L=18.1-18.7 \mathrm{~cm}^{-2}$ (where $T_{\text {abs }}$ is the temperature of maximum absorption). Assuming axi-symmetry along the direction of propagation, the LOS depth across the clump is then roughly equal to the clump's width. The clump's width in the EUV filters is 2." 8 , although it cannot be determined with precision due to the poor spatial resolution of AIA. The clump in $\mathrm{H} \alpha$ has a maximum width of $0 . " 6$ (with an average of $0 . " 4$ ). From the results of data set 2 , it is likely that the larger widths in EUV are mostly an effect of the lack of resolution (which would smear out the width a factor proportional to AIA's PSF). We therefore assume an EUV width 1.5-2 times larger than the $\mathrm{H} \alpha$ width, leading to a width of roughly $700 \mathrm{~km}$. This leads to an electron density of $1.8-7.1 \times 10^{10} \mathrm{~cm}^{-3}$, where the scatter is most likely due to the presence of inhomogeneities within the clump. This is supported by the fact that the spectral width of the clump in $\mathrm{H} \alpha$ indicates a temperature of $5500 \pm 500 \mathrm{~K}$, suggesting a multi-temperature structure, and possibly a higher density. Assuming a constant pressure within the clump the core density can then be up to $2.5 \times 10^{11} \mathrm{~cm}^{-3}$.

\subsection{Clumpy Versus Continuous}

As the clumps fall further down toward the sunspot in data set 1 they become absorption features in the wing of $\mathrm{H} \alpha$ while they gradually become bright toward line center (see Figures 4 and 5). These distinctive features allow us to trace them down to chromospheric levels, just before impact. Similarly as previously done for coronal heights we define two cuts at chromospheric heights roughly perpendicular to the clumps trajectories, F1 and F2, corresponding to loops 1 and 2, respectively. Both $\mathrm{C}$ and $\mathrm{F}$ pairs are plotted in dashed curves in Figure 5. As in Animations 3 and 4 for $\mathrm{C} 1$ and $\mathrm{C} 2$, Animations 5 and 6 show the detection of rain clumps across F1 and F2, respectively (in red the FWHM of the fit to the clump's width). These animations indicate that the $\mathrm{C} 1$ clumps fall either into the umbra or toward the closer edge of the sunspot (projection effects do not allow us to firmly pinpoint the location of the fall) and the $\mathrm{C} 2$ clumps seem to fall mainly toward the far edge of the sunspot. Tracing of coronal rain clumps to such low heights has never been achieved before and is possible here thanks to the high falling speeds of the clumps (which produces the dark absorption features in the wing of $\mathrm{H} \alpha$ ) and to the high spatial and temporal resolution of CRISP. Indeed, the total velocities (taking into account projected and Doppler velocities) vary between 80 and $120 \mathrm{~km} \mathrm{~s}^{-1}$ for both loops.

Due to the increase of projection effects at low heights, the high speed of the clumps and the seeing effects (which do not alway provide clear subsequent images), it is not possible in most cases to specify the start and end for a clump at low heights, and therefore provide a robust determination of clump lengths. It is therefore possible that two subsequent detections at the same location correspond to the same clump. Also, it is highly likely that many of the clumps detected at heights $\mathrm{C}$ are detected again at heights $\mathrm{F}$. However, since we are more interested in regarding the rain as a flow rather than a discrete set of condensations (especially because their morphology can change dramatically as they fall), for our statistical analysis we consider that all clumps are different (and for the lengths we give an approximate value determined visually). Since there are many clumps that fail to be detected by the algorithm (due to 


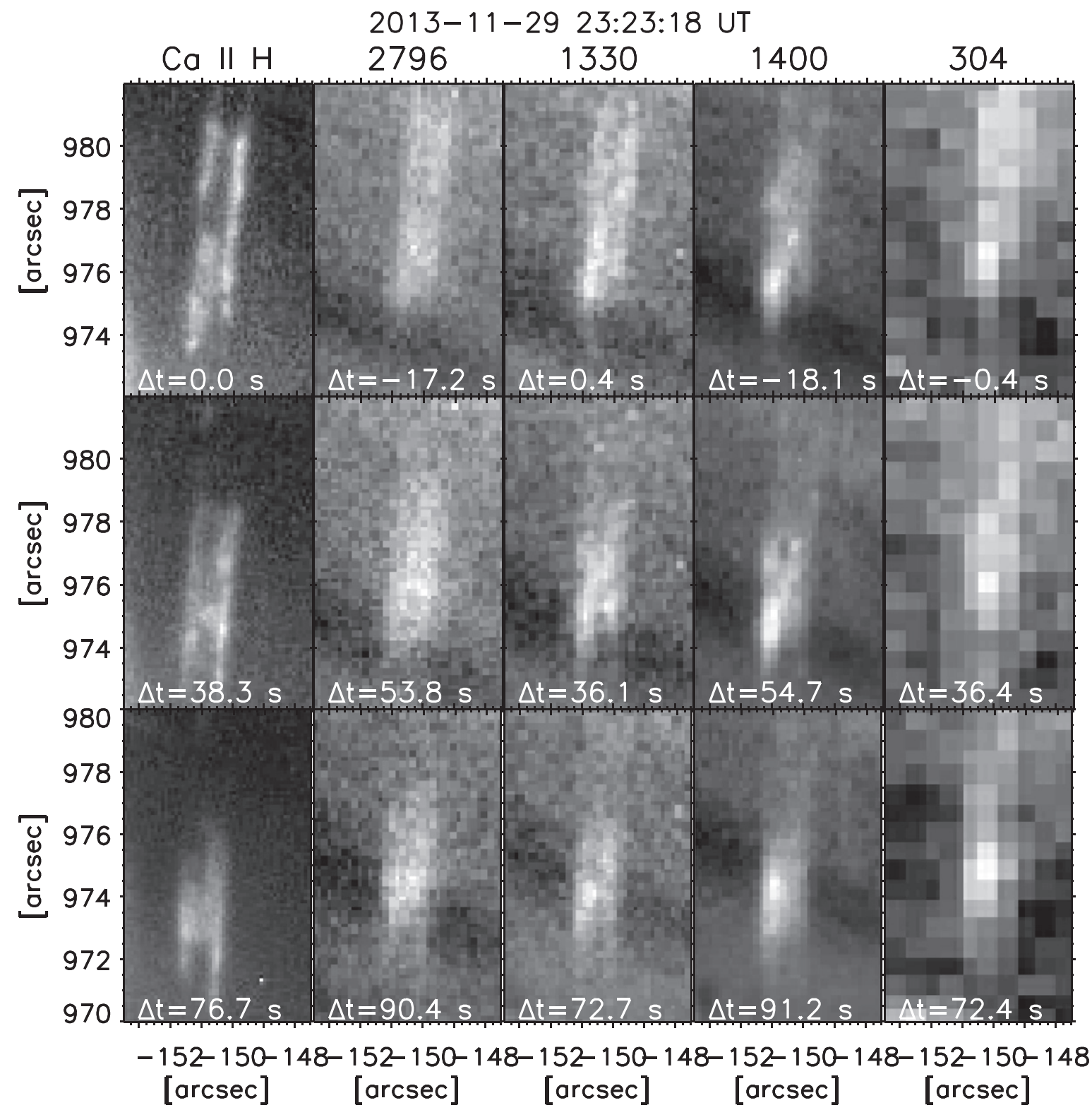

Figure 11. Substructure for a rain shower in the rain event of data set 2 . The shower corresponds to the head of the rain and is shown at three different times separated by roughly $38 \mathrm{~s}$ from each other (shown in the rows) and for different wavelengths (shown in the columns). From left to right we have Hinode/SOT in Ca II H, IRIS/ SJI 2796, 1330, 1400, and SDO/AIA 304. The SJI and AIA snapshots correspond to those closest in time to the SOT snapshot in the first column of each row. The time difference with respect to the first snapshot (top left panel) is indicated in the bottom of each panel.

the previously stated reasons), we consider that this procedure leads to a better approximation to the real rain population.

A striking feature shown by Animations 5 and 6 is that at such low chromospheric heights the clumps appear significantly less clumpy (their lengths increase) and the downward flow becomes continuous and rather persistent. In order to quantify this effect better we plot in Figure 13 a histogram of clump detection weighted with their respective widths squared at the two previously defined heights for loops 1 and 2. Not only are the clumps in the corona detected at later times in the chromosphere, but we also detect many more rain crossings at those lower heights, indicating an increase in the rain flow with decreasing height (46 and 145 clumps for $\mathrm{C} 1$ and $\mathrm{F} 1$, respectively; 95 and 193 clumps for $\mathrm{C} 2$ and F2, respectively). In Section 5.4 we discuss possible reasons for this increase in clump occurrence at low chromospheric heights.

\subsection{Strand-like Structure}

As shown in Section 4.2, the presence of substructure in coronal rain at high resolution is not only clear at chromospheric temperatures such as those of $\mathrm{Ca}$ II $\mathrm{H}$ and SJI 2796 but also at higher temperatures, such as those of SJI 1330 and SJI 1400. As shown in Figure 11, as the rain falls substructure is observed to be organized along strands of various lengths but rather similar widths. This is also observed in Animation 7 for the rest of the rain in this event and in data set 1 at even higher resolution. Indeed, at the highest resolution achieved in this work (in $\mathrm{H} \alpha$ with CRISP) similar strand-like structure appears, as shown in Figure 4 and Animations 3-6. Due to the very dynamical nature of coronal rain it is not easy to discern the strand-like structure traced in time by the rain. For this, we plot in Figure 14 the time variance in a Doppler image in $\mathrm{H} \alpha \pm 0.6 \AA$ over a time period of $22 \mathrm{~min}$ for loop 1 and its 


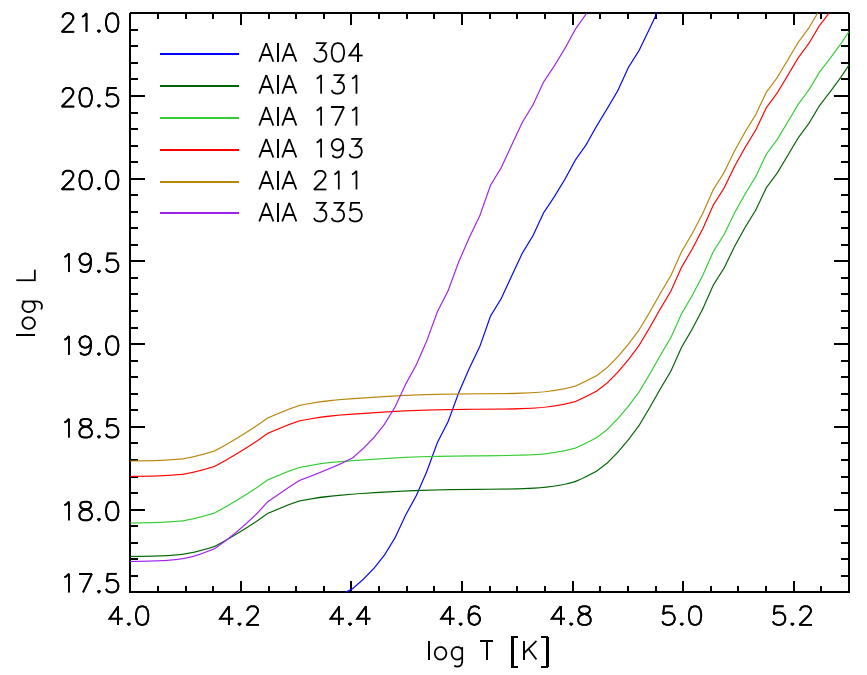

Figure 12. $L$ function (in logarithmic scale), as defined in Landi \& Reale (2013), with respect to temperature, for the various AIA filters calculated over the trajectory of a rain condensation producing EUV darkening.

\section{H $\alpha$ rain occurrence in corona and chromosphere}

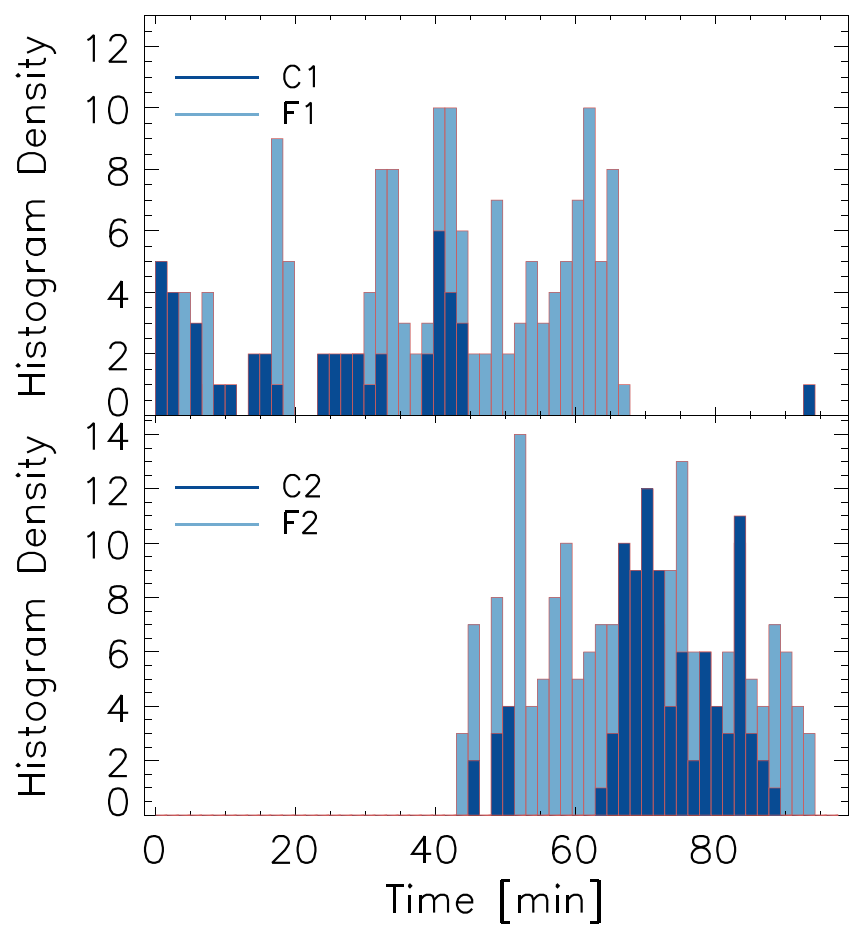

Figure 13. Histograms of rain condensations detected in $\mathrm{H} \alpha$ with SST/CRISP for data set 1 along cuts $\mathrm{C} 1, \mathrm{~F} 1$ and $\mathrm{C} 2, \mathrm{~F} 2$ for loops 1 and 2, respectively. The condensations detected at coronal heights ( $\mathrm{C}$ cuts) constitute the deep blue histogram, while those detected at chromospheric heights (F cuts) constitute the light blue histogram. The histograms are constructed by taking a bin size of a $100 \mathrm{~s}$, and for each time bin the intensity of all contained clumps are summed, each multiplied by the clump's width squared.

surroundings (where the time variance is defined as the sum of the squared average-subtracted intensity for each pixel). Thanks to the emission features of the rain in the wing of $\mathrm{H} \alpha$ both above the spicular layer and at chromospheric levels, the clumps produce bright traces in the variance image. These traces appear as various strands at both of these heights (in the

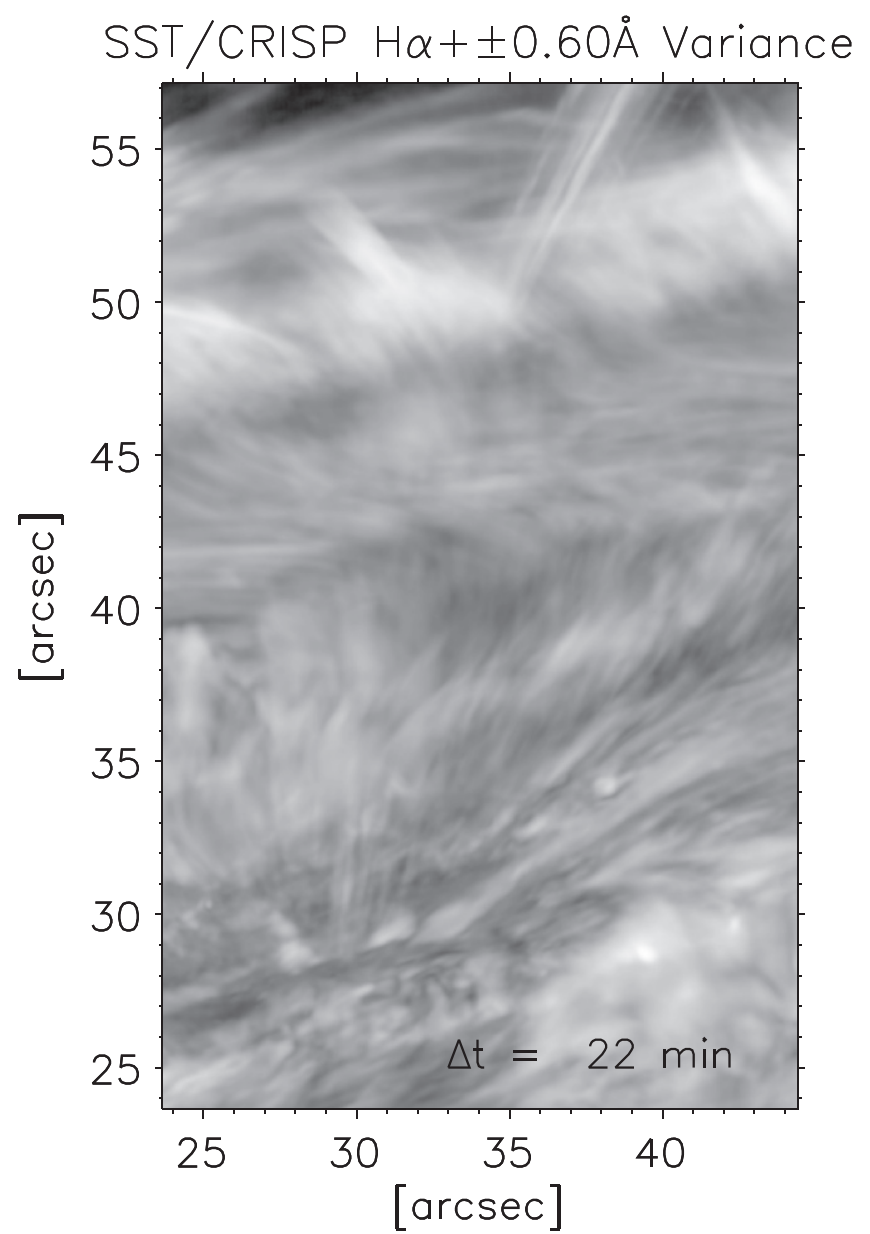

Figure 14. Variance of a Doppler image in $\mathrm{H} \alpha \pm 0.6 \AA$ with SST/CRISP over a time of $22 \mathrm{~min}$ for loop 1 . Various strands are visible from $50^{\prime \prime}$ to $55^{\prime \prime}$ and $28^{\prime \prime}$ to $35^{\prime \prime}$ in the vertical axis.

figure, these are visible from $50^{\prime \prime}$ to $55^{\prime \prime}$ and $28^{\prime \prime}$ to $35^{\prime \prime}$ in the vertical axis). In the figure, 6-8 strands with widths around $0 .{ }^{\prime \prime} 2-0 . " 4$ can be discerned at both heights, although a larger number may exist due to projection effects. It is worth noting that the 22 minute period over which the variance is taken is a significantly long time for coronal rain. Indeed, the number of clumps detected over this time period is on the order of 60-70. Also, as calculated previously in Section 3.2, the travel time for rain in these loops is on the order of $20 \pm 10 \mathrm{~min}$. Last, numerical simulations indicate that the catastrophic cooling part of limit cycles is generally short, under an hour or so (Mendoza-Briceño et al. 2005; Antolin et al. 2010; Susino et al. 2010; although this depends on various parameters of the heating). Therefore a significant part of the catastrophic cooling for this loop may be captured in the time variance, strongly suggesting that the clumps follow well-defined fundamental magnetic structures in the corona.

Interestingly, apart from the prominent clumps as those in Figure 11, the surrounding diffuse atmosphere in data set 1, dimly bright in $\mathrm{H} \alpha$, also appears structured in the same way. This is especially clear at times of large clumps. An example of this is shown in Figure 15, where a snapshot of a falling clump can be seen in $\mathrm{H} \alpha \pm 1.1 \AA$ A. A bright clump with a long tail can be seen and, particularly, multiple strand-like structures next to it, as ripples with similar widths down to $0 . " 15$ or so, uniformly distributed within $4 "$ of the clump. This structure can still be 

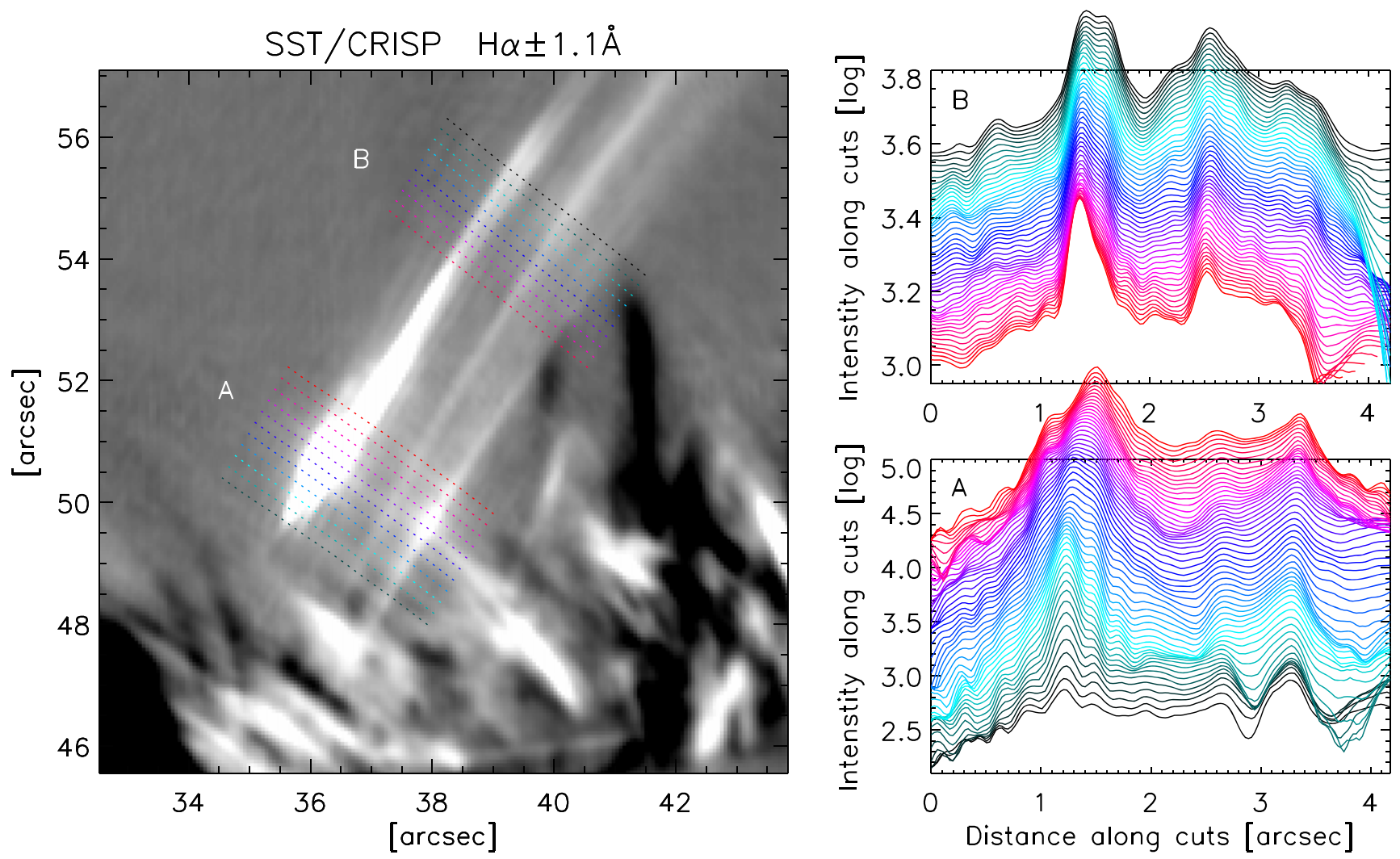

Figure 15. Rain condensation captured in H $\alpha$ by SST/CRISP, here shown as a Doppler image (left panel) at $\pm 1.1 \AA$. The intensity of the image is saturated in order to better visualise the small dim structure parallel to the main condensation. Transverse cuts to the direction of propagation of the condensation are shown toward the head (location A) and tail (location B). The intensity along these cuts is shown in the right panels at both A and B locations as stack curves separated by an ad hoc value. The color of the intensity curve corresponds to the cut of the same color in the left panel (for location A or B).

discerned (although with decreasing intensity) when shifting the wavelength position over a range of $\pm 0.5 \AA$ around $1.1 \AA$, ruling out possible instrumental effects from the narrowband CRISP wavelength channels. In order to show the strand structure better we make transverse cuts across the clump at two different locations, one at the head and one at the tail of the clump. The $\mathrm{H} \alpha$ intensity along these cuts is shown in the right panels of the figure. Next to the large peak corresponding to the bright clump, around 8 ripples can be distinguished extending significantly along the direction of propagation. This structure is highly reminiscent of the MHD thermal mode (also known as the entropy mode in the absence of thermal conduction), first predicted by Field (1965) and later developed by van der Linden \& Goossens (1991a, 1991b), Goedbloed \& Poedts (2004), Murawski et al. (2011). Indeed, the spatial distribution of such mode is that of a main elongated dense clump with multiple smaller clumps side by side of similar widths. The generation of this wave is guaranteed by the small but non-zero perpendicular thermal conduction in the corona. Although static in an ideal scenario, this wave is expected to move together with the flow. In this scenario, the observed strand-like structure at the smallest detected scales could be the result of MHD thermal modes produced from thermal instability.

\subsection{Sizes}

We now turn to the statistical determination of sizes. The methods used in the widths and lengths calculations are explained in Section 2.3. The routines used for this detection are semi-automatic. The detection of a clump is rather strict, and is based on several conditions which rule out most of the fainter and smaller clumps at diffraction limit resolution. For instance, the smaller widths that can be discerned in the cross profiles in Figure 15 are mostly left out by the algorithm, in favor of precision and reduction of errors.

In Figure 16 histograms displaying the relative frequency (for each wavelength) for the obtained widths and lengths are shown, combining data sets 1 and 2, and therefore all the instruments used in this study (note that the bin widths of the distributions have been set equal to the resolution of each instrument). Due to the large number we restrict the histograms to the more interesting small scales part (upper panels) and separate between the high (CRISP, SOT, SJI) and low resolution instruments (AIA in the lower panels). The figure clearly shows a significant difference between widths and lengths at all wavelengths. The widths appear nicely distributed, with increasingly higher occurrence frequency at smaller scales for each wavelength and a tail at longer scales. Such distribution shape seems therefore independent of temperature, with an apparent peak for all clumps between $0 .{ }^{\prime \prime} 2$ and 1." $1 . \mathrm{H} \alpha$ and $\mathrm{Ca}$ II $\mathrm{H}$ clump distributions have, respectively, narrow and broad peaks at 0.25 and 0." 6 . Blobs observed with IRIS in SJI 2796, SJI 1330 and SJI 1400 have similar distributions peaking at $0 .{ }^{\prime \prime} 6-0 .{ }^{\prime \prime} 8$. We note, however, that SJI 2796 presents a broader distribution for the peak, between $0 . " 6$ and 1 ", probably due to the higher opacity compared to the optically thinner $\mathrm{C}_{\mathrm{II}}$ and $\mathrm{Si}$ Iv lines. For AIA we note that while widths in 171 and 193 peak around $1 . " 8-3$, 304 peaks around $0 . " 8-1.7$. 

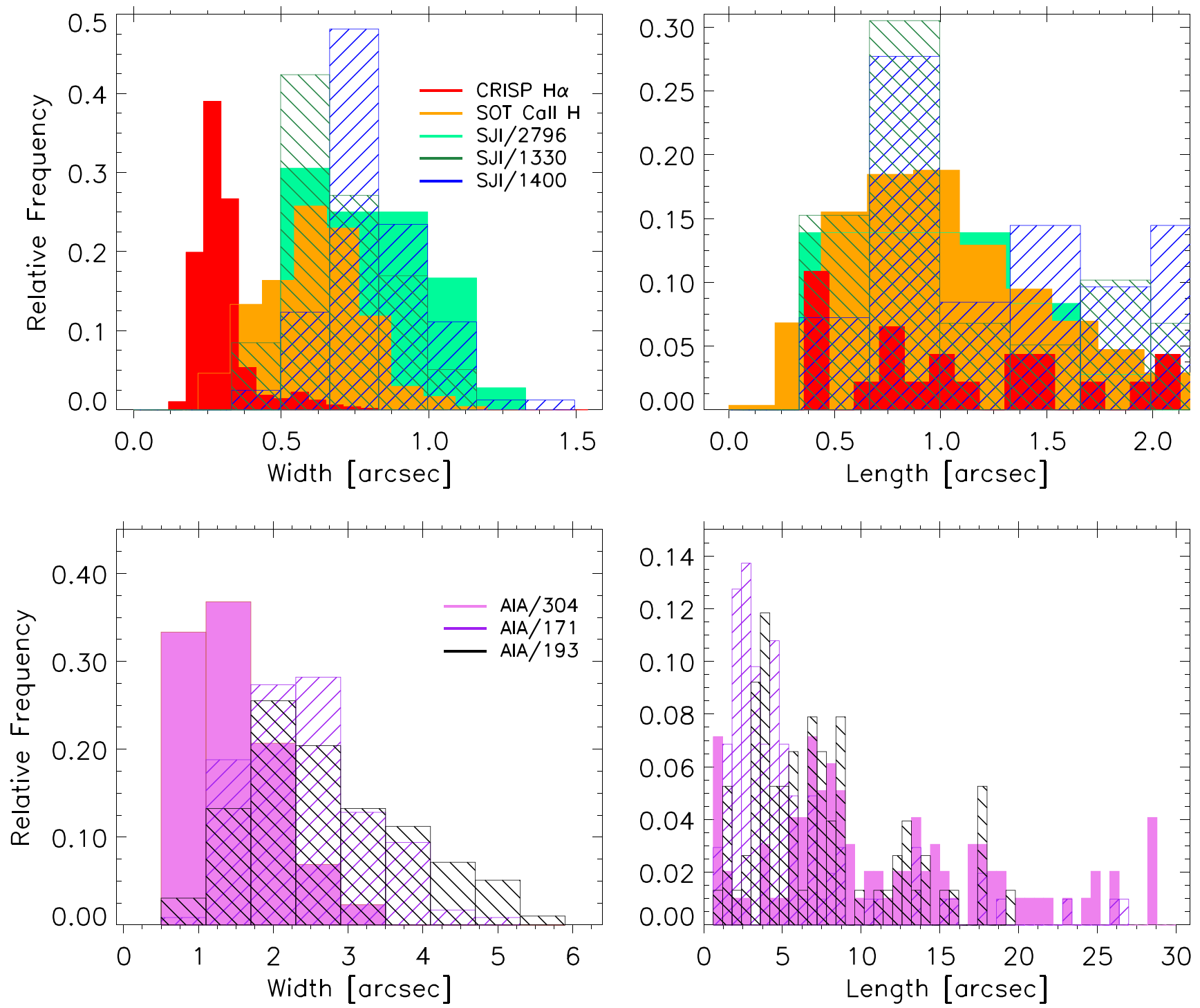

Figure 16. Histograms of calculated widths (left) and lengths (right) for the detected coronal rain condensations in the various wavelengths used in the present study for data sets 1 and 2. The corresponding color for each wavelength is shown in the legend. The bin size in each wavelength is set to the spatial resolution of each instrument. For the high resolution instruments (upper panels) only the small scale part of the spatial range is shown. In the lower panels only the AIA results are shown.

On the other hand, the lengths show a more random distribution, although with high clustering at small numbers, between $0 . " 5$ and $1 . .5$. It is worth noting that the lengths distribution spans up to $30^{\prime \prime}$ or so (for instance, the very long clump of Figure 4). For lengths longer than 5" (not shown here) the distribution becomes sparse.

In Table 1 average values for each wavelength and data set are shown, together with the total number of clumps detected in the FOV per minute as an indication for the number of clumps detected in each data set. We choose to calculate this number since a clump is a not well-defined plasma structure over time. As shown in the present animations (and in Figure 11) and in Paper I, clumps can dramatically change shape, appear and disappear along their fall. This number needs to be read with caution: the area over which rain is detected in data set 1 is roughly half that in data set 2 (therefore, dividing the number over area would lead to a similar number between SST and Hinode). The continuous flow in 304 (as opposed to clumpy) also makes it difficult to identify a clump. For AIA only clear cut examples of clumps were selected, which explains the low number per minute.

The widths presented here in $\mathrm{H} \alpha$ are similar to previously measured widths in the same wavelength with CRISP in large statistical data sets (Antolin \& Rouppe van der Voort 2012; Antolin et al. 2012), but present a relatively higher number of clumps at lower spatial scales. This is mainly due to the fact that the CRISP instrument gained from improved spatial sampling after an upgrade performed in 2009. The widths of $\mathrm{Ca}$ II $\mathrm{H}$ clumps are similar to those reported in Antolin et al. (2010). In the top left panel of Figure 16 the tendency of number increase for clumps with smaller widths suggests a tipof-the-iceberg scenario.

As expressed previously, the lengths appear to be more randomly distributed, especially in $\mathrm{H} \alpha$, and therefore may be more data set dependent. For instance, extremely long clumps are only observed in data set 1 , and have not been previously 
Table 1

Average Values for Both Data Sets

\begin{tabular}{lcccc}
\hline \hline $\begin{array}{l}\text { Instrument } \\
\text { and Filter }\end{array}$ & Data Set & $\begin{array}{c}\text { Width } \\
\text { (arcseconds) }\end{array}$ & $\begin{array}{c}\text { Length } \\
\text { (arcseconds) }\end{array}$ & $\begin{array}{c}\text { Blobs } \\
(/ \text { minutes })\end{array}$ \\
\hline CRISP H $\alpha$ & 1 & $0.3 \pm 0.093$ & $3.85 \pm 5.77$ & 5. \\
SOT Ca II H & 2 & $0.61 \pm 0.17$ & $1.05 \pm 0.51$ & 9.7 \\
SJI 2796 & 2 & $0.8 \pm 0.19$ & $2.49 \pm 1.97$ & 2.6 \\
SJI 1330 & 2 & $0.7 \pm 0.15$ & $1.75 \pm 1.35$ & 3.7 \\
SJI 1400 & 2 & $0.8 \pm 0.17$ & $1.76 \pm 1.26$ & 4.6 \\
AIA 304 & 2 & $1.56 \pm 0.15$ & $10.8 \pm 6.5$ & 2.2 \\
AIA 304 & 1 & $1.4 \pm 0.7$ & $12.7 \pm 9.2$ & 1.2 \\
AIA 171 & 1 & $2.45 \pm 0.81$ & $5.5 \pm 5.2$ & 1.2 \\
AIA 193 & 1 & $2.69 \pm 1.07$ & $7.4 \pm 4.6$ & 1.2 \\
\hline
\end{tabular}

Note. Averages are calculated over all detected blobs in both data sets. Row order is set according to increasing temperature. For data set 1 the measurements are limited only to the rain detected in loops 1 and 2. For data set 2 the measurements are limited to the loop shown in Figure 8. While each measurement is itself an average value over a large quantity (see Section 2.3) and therefore has its own standard deviation, the standard deviation shown here for widths (lengths) is simply taken over the set of blob widths (lengths). The last column provides the number of blobs detected per minute for each data set, which implies dependence over the FOV over which this detection is carried. For the SST, the detection is done over a small part of its FOV (roughly $5^{\prime \prime} \times 17^{\prime \prime}$ ), which is much smaller than the FOV for the SDO data of the same data set (see Figure 3). For Hinode the projected area over which the the rain is detected is roughly twice that of SST.

observed in other data sets in $\mathrm{H} \alpha$ with CRISP, except in the recent work by Scullion et al. (2014) in the context of flares, i.e., stronger footpoint heating sources.

\section{DISCUSSION}

\subsection{EUV Intensity Variations as a Signature of Catastrophic Cooling}

Coronal loops in active regions often present intensity variations, which are regularly associated with states out of hydrostatic equilibrium such as states of thermal nonequilibrium (Aschwanden et al. 2001; Reale 2010). A state of thermal non-equilibrium implies the presence of thermal instability in coronal loops. This instability can be thought as the most general state of the plasma (meaning that thermal equilibrium is extremely unlikely). The relevant question here is what are the timescales of this instability in coronal loops? Accordingly, this instability can have a complete character, in the sense that runaway cooling due to the shape of the optically thin loss function may occur locally in coronal loops and continue uninterrupted down to chromospheric temperatures, until heating from local sources overcome the losses due to its own radiation. The completeness of thermal instability, however, is not guaranteed and depends strongly on the efficiency of the heating mechanisms and on thermal conduction. Mikić et al. (2013) have shown that even changes in geometry (non-uniformity in area cross-section and asymmetry in the heating) play an important role in determining the aspect of this instability. Furthermore, the spatial scales involved in the cooling processes (for instance, the clumpiness and strandlike structure of the rain) are directly related to the properties of the plasma and to the counter-acting heating mechanisms, and may therefore reveal essential characteristics of the heating processes.
Despite the close link between thermal instability and coronal heating and therefore the importance of thermal instability, we do not know how ubiquitous this instability is in the corona and the details of its character (for instance, whether it is complete or not). This is largely due to the high complexity of the thermal instability in strongly anisotropic plasmas such as the solar corona. Recent observational studies over active regions indicate a general tendency for cooling (Viall \& Klimchuk 2012). Although it is clear that not all the loops in an active region are in a thermal non-equilibrium state and undergo catastrophic cooling, it is important to estimate what fraction of loops are.

The traditional picture of thermal instability implies that coronal loops in a thermal non-equilibrium state become progressively cooler, suggesting a time delay between filters detecting emission at different temperatures. Such cooling progression throughout TR temperatures has previously been invoked to explain variations in EUV light curves (Foukal 1976, 1978; Kjeldseth-Moe \& Brekke 1998; Schrijver 2001; O'Shea et al. 2007; Warren et al. 2007; Landi et al. 2009; Tripathi et al. 2009; Ugarte-Urra et al. 2009, 2006; Kamio et al. 2011). However, the direct link to catastrophic cooling has not been yet firmly established, since all of these multi-wavelength observations do not include chromospheric ranges. In this work we make the first steps in this direction by linking commonly found intensity variations in EUV filters of SDO/AIA to the observational imprint of catastrophic cooling, i.e., coronal rain observed in chromospheric diagnostics.

We observe complete thermal instability with temperatures down to the chromospheric range. In Section 3 we show that EUV darkening is found to be associated with flows in AIA 304 and presents a continuous and rather persistent character. Such AIA 304 flows are observed along many of the loops within the active region (data set 1), part of which are captured toward the footpoints in H $\alpha$ by CRISP. The flows in AIA 304 appear strongly correlated in space to the appearance of cool chromospheric material in the loops observed in $\mathrm{H} \alpha$, which occurs in a more intermittent way. This suggests that while the entire loop may be thermally unstable and cooling, plasmas at TR temperatures are more widespread and common within the loop than plasmas at chromospheric temperatures. This is expected to some extent due to the tendency of the plasma to keep a uniform pressure distribution along the field (and the fact that thermal instability entails a local loss of pressure leading to small condensations).

Small EUV intensity perturbations are further strongly correlated in time with the presence of $\mathrm{H} \alpha$ rain clumps. Although intermittent, these clumps can come in series of events and generate quasi-periodic EUV intensity variations on the order of a few minutes. In most cases, these variations are found to be associated with groups of chromospheric clumps which we have previously termed "showers" (Paper I), and span over a few arcseconds in the transverse direction. Correlation between EUV darkening and $\mathrm{H} \alpha$ has been extensively studied in the case of prominences (Heinzel \& Anzer 2006; Labrosse et al. 2010). For the EUV wavelengths of interest here, this correlation is mainly due to continuum absorption from neutral hydrogen, neutral helium, and singly ionized helium. 

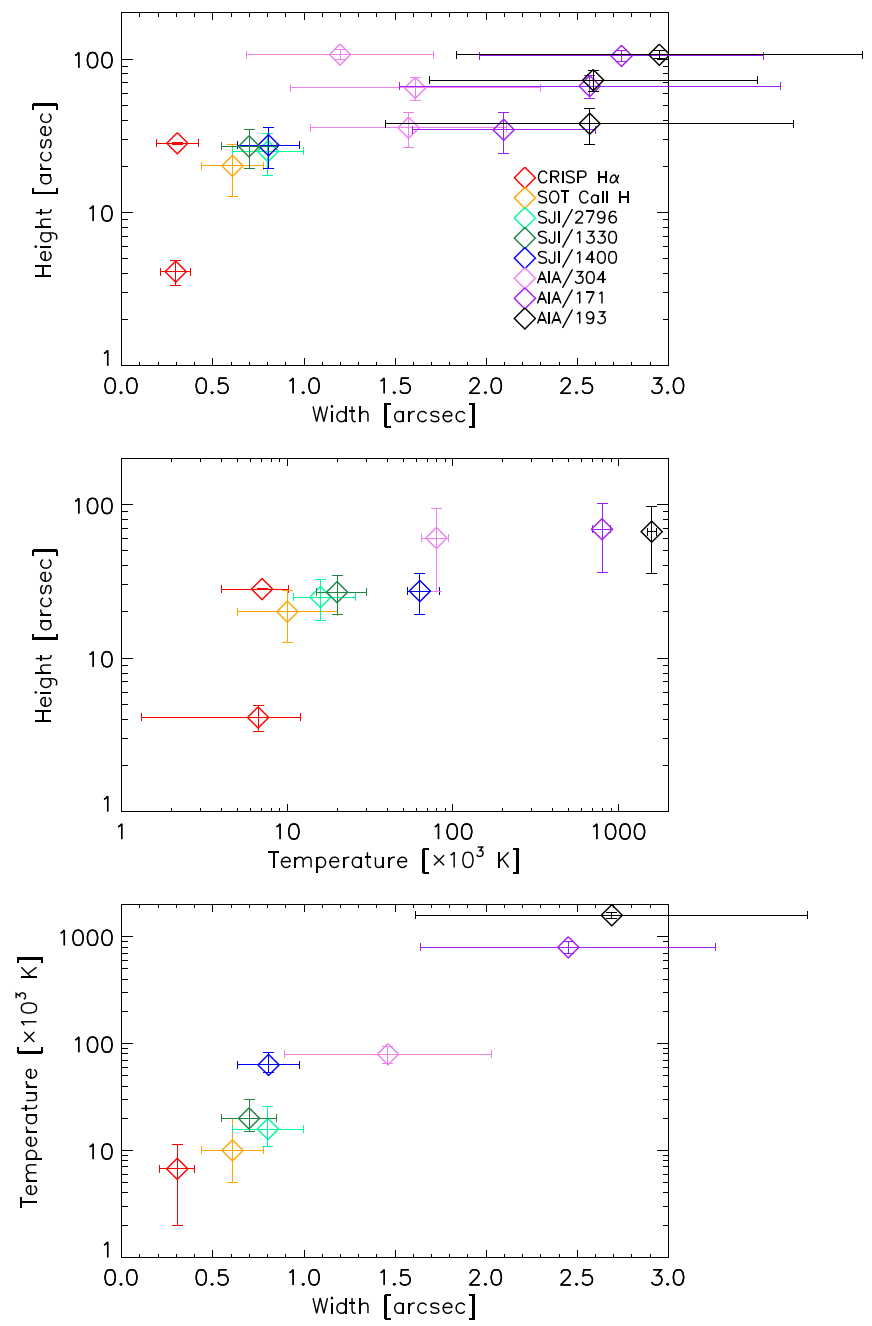

Figure 17. Diagrams showing relationship between various quantities. As quantities we have the height of the measurements, estimated from the footpoint location of the loop in the chromosphere, the width of the condensations and the temperature of the condensations. For each quantity $Q$, its average value $\langle Q\rangle$ over the entire set of measurements is plotted, together with error bars corresponding to the standard deviation $\sigma(\langle Q\rangle \pm \sigma)$. Each color corresponds to a different wavelength, indicated in the top panel.

\subsection{Multithermal Character}

The progressive cooling picture from thermal instability also suggests a difference in emission with height. In this picture, emission in $\mathrm{H} \alpha$ and at cooler wavelengths is preferentially detected at low coronal heights. In the middle panel of Figure 17 we plot the averages of height versus temperature for all detected clumps in data sets 1 and 2 . The temperatures taken for the SJI and SOT filters are those of maximum formation for the dominant ions of each filter. Those for CRISP correspond to the measured temperatures, shown in Figure 6. Although not strongly correlated, the plot suggests a slight temperature decrease with height. Particularly, the very low temperatures below $5000 \mathrm{~K}$, even down to $2000 \mathrm{~K}$ or so, for the H $\alpha$ clumps detected with CRISP at the lower part of the loops provides support to the cooling picture.

Through co-observation between IRIS and Hinode/SOT we are able to follow for the first time the rapid progression in the runaway cooling in thermally unstable loops. A two-step progression is found in which fast sudden appearance at TR and upper chromospheric temperatures is followed by a more gradual appearance at lower chromospheric temperatures. This two-step scenario agrees with the shape of the optically thin loss function, which predicts runaway cooling down to $10^{5} \mathrm{~K}$ or so, below which material becomes progressively optically thick, and therefore radiation has a lower escape probability (leading to less efficient radiative cooling).

Despite the progressive cooling from transition temperatures to chromospheric temperatures found in data set 2 the AIA 171 intensity along the loop is found to increase about $10 \mathrm{~min}$ prior to the rain event, and remains bright and even further increases at the end, during the rain event. Such behavior strongly suggests the presence of multithermal plasma in the loop, from chromospheric to coronal temperatures. Furthermore, it implies that the progressive cooling picture down from coronal temperatures to chromospheric temperatures is not always reflected in the evolution of EUV light curves. Hot material can co-exist with catastrophically cooling plasma. Therefore a hot to cool systematic sequence in EUV channels is not an observable requirement for a loop in a thermal nonequilibrium state.

The obtained cooling down to chromospheric temperatures is very fast, achieved in a timescale that varies from tens of minutes (data set 1 ) down to minutes (data set 2 ). Due partly to this reason, in both analyzed data sets, and especially in the one analyzed with SJI and SOT, thanks to the high spatial and temporal resolution, we find a high degree of co-spatiality in the multi-wavelength emission over the entire span of the event. This strongly supports the presence of multithermal plasma in these loops. Furthermore, the multithermal character is accompanied by strong density inhomogeneity, which suggests a complex thermodynamic evolution within the same loop. The multithermal picture is also supported by the results of Scullion et al. (2014), which show $\mathrm{H} \alpha$ emission with CRISP co-located with EUV AIA 171 in post-flare loops.

The large range of temperatures we detect imply that coronal rain, as a result of catastrophic cooling, is not solely a chromospheric phenomenon but a TR phenomenon as well. Actually, since it is material cooling from coronal temperatures and, as discussed previously, it is more pervasive in TR temperatures, it is mainly a TR phenomenon which spans down to chromospheric temperatures if the thermal instability is complete. This result matches well the picture that is being perceived with increasing frequency in SJI and AIA synoptic observations, namely, that basically every active region shows rain-like phenomena in AIA 304 and SJI 1400. A proper statistical analysis of this picture is left as future work.

For data set 1, the EUV emission (darkening or brightening) in AIA 304 is found to be wider than the chromospheric emission in $\mathrm{H} \alpha$ or $\mathrm{Ca}$ II $\mathrm{H}$ suggesting a wide transition from chromospheric to TR temperatures. However, the highly cospatial emission obtained from SJI and SOT in data set 2 suggests that the wide transition in data set 1 is mainly due to the lack of resolution in AIA. The observed thin transition in data set 2 must exist on spatial scales below the resolution of $I R I S$, i.e., 0."33, which is also supported by the similar width distributions obtained with SOT and the SJI filters (see Figure 16). Indeed, when closely comparing the substructure of clumps, small structural differences appear between the clumps in Ca II $\mathrm{H}$ and in SJI 2796, 1330, and 1400. This multithermal plasma picture and the large-density inhomogeneities at such high resolution suggests that the thermal instability mechanism in a strongly anisotropic magnetic field such as the 
corona is far more complex than the simple picture of a uniformly progressive cooling plasma with cool chromospheric cores surrounded by warmer diffuse material. On the other hand, the difference in the average widths between rain in emission in AIA 304 and rain in absorption in AIA 171 and AIA 193 suggests a relatively wide transition from TR to coronal temperatures on the order of 0.5 (known as the Prominence Corona Transition Region, PCTR, in the case of prominences; Chiuderi \& Chiuderi Drago 1991; Heinzel et al. 2001), similar to reported values in prominences (Vial et al. 2012; Parenti 2015). This suggests a large increase in complexity in the interface between chromospheric and TR plasmas, and less so for the PCTR. It is also possible, although less likely, that since absorption in the AIA 171 filter is produced by multiple elements (but mainly $\mathrm{H}, \mathrm{He}$, and $\mathrm{He}^{+}$), an inhomogeneous He distribution within the loops (more concentrated in the core of the loops than hydrogen) could lead to such difference in widths. How such a high degree of inhomogeneity, especially at the smallest scales, is achieved in such loops is an important question that needs to be addressed in future work. Theoretically, the presence of high degree complexity at the smallest scales could be explained by the existence of tangential discontinuities in the field in which material can collapse, predicted by stability analysis of prominence plasma in similar conditions as in the present observations (Low et al. 2012a). Such theoretical studies also predict the constant evolution of magnetic fields and fluid at these small scales leading to spontaneous formation and resistive dissipation of discrete currents (Low et al. 2012b). Such events may explain the constant morphological and physical changes of coronal rain clumps that we observe along their fall, also reported in Harra et al. (2014).

\subsection{Elemental Scales and Global Topology}

The present results strongly suggest that the bulk of the distribution is below the highest resolution used here $\left(\approx 0.0^{\prime \prime}\right)$. This study confirms and extends the result of Scullion et al. (2014) over various instruments spanning different temperatures. This picture is also obtained in 2.5D MHD simulations of coronal rain by Fang et al. (2013), in which the number of rain clumps keeps increasing at seemingly ever decreasing scales. In Figures 16 and 17 (upper panel) the standard deviation of widths and the ratio between standard deviation and mean values for the widths decreases significantly from the AIA measurements down to CRISP measurements (except for the AIA 304 widths in the second data set, perhaps reflecting the absence of large-scale coronal rain downflows and no significant showers in this case). On the other hand, the lengths present a more random distribution with peaks at a few arcseconds or less, which is also found in numerical simulations by Luna et al. (2012) and Fang et al. (2013). We further find extremely long clumps encompassing a few tens of thousands of kilometers. This large scatter in lengths may reflect the presence of several factors at play along the field (such as flows, thermal conduction, instabilities, field geometry, etc.), supported by numerical results. The more uniform distribution of widths, the agreement over a significant number of data sets (Antolin \& Rouppe van der Voort 2012; Antolin et al. 2012; Harra et al. 2014; Scullion et al. 2014) and the similarity with widths of prominence threads (Lin et al. 2005) suggests a more fundamental mechanism behind the shaping of condensations in the transverse direction to the field. This behavior strongly suggests the existence of elemental strandlike structures at smaller scales. Such fundamental scales would be defined by a similar thermodynamic and magnetic field evolution. A relevant question to ask is then whether thermal instability plays a main role in the shaping of such structures, and especially defining the widths of these elemental structures, or whether the obtained widths would also be expected for substructure in a thermally stable loop. In Figure 16 spatial resolution and spectral diagnostic (and therefore temperature) are intertwined in the results and it is difficult to disentangle them and estimate the influence of each on the observed widths. The relation between temperature and width is shown in the lower panel of Figure 17, where we can see a roughly monotonically increasing trend. However, the fact that $\mathrm{H} \alpha$, $\mathrm{Ca}$ II $\mathrm{H}$, and $\mathrm{Mg}$ II $\mathrm{k}$ form at roughly similar chromospheric temperatures but show a significant difference in widths points mostly to a major role of resolution over temperature (although less so from $\mathrm{Mg}$ II $\mathrm{k}$ due to the higher opacity). Also, the widths found in $\mathrm{C}_{\mathrm{II}}$ and especially in Si IV are similar, despite the significant difference in temperature formation, suggesting that such small strand-like structures may extend to hotter lines at high spatial resolution.

It is nonetheless possible that the observed strand-like structure in these loops, or part of it, is the product of thermal instability. For instance, in Section 4.5 we showed at the smallest scales we may have an influence of the MHD thermal mode on the shaping of strand-like structure. The ripples in the clump's neighborhood generated by this wave imply a strandlike structure all around the main clump. These ripples constitute small density enhancements (which correspond to the spatial shape of the thermal mode eigenfunctions) that propagate together with the clump and can enhance radiative losses. It is therefore likely that such perturbations may grow into condensations themselves, leading to further strand generation (Soler et al. 2012). Besides this effect, catastrophic cooling can also introduce large pressure variation, especially in the strong density inhomogeneities found here with cool cores with electron densities on the order of $10^{11} \mathrm{~cm}^{-3}$. Due to flux freezing the collapse of material following thermal instability generates an increase of the magnetic field. Despite the local character of the condensation the subsequent field enhancement is non-local due to the rigidity of the coronal magnetic field (high magnetic tension due to the high external Alfvén speed, An 1984). This process can thus generate a (slightly) magnetically different strand which would in turn shape warmer material up- and downstream. Such a scenario has been obtained in two-dimensional MHD simulations of thermal instability in the interstellar medium, with similar plasma $\beta$ values (Sharma et al. 2010). This scenario implies a relation between clumps' widths and the magnetic field at small scales, which offers interesting coronal seismology applications.

The proposed non-local enhancement of the magnetic field would increase the magnetic pressure downstream of the clumps, which could explain the less than freefall speeds (and rather constant accelerations) always observed in coronal rain. Let us make an order-of-magnitude estimate of this possibility and leave a detailed analysis for future work. Let us consider the case of data set 1 for which we have density estimates. We have an average velocity at the footpoint of $100 \mathrm{~km} \mathrm{~s}^{-1}$, a loop height of $42 \mathrm{Mm}$, and let us assume a negligible initial speed at the apex of the loop. The average effective gravity along an 
elliptic loop with a ratio of loop height to half baseline between 0.5 and 2 varies between 0.132 and $0.216 \mathrm{~km} \mathrm{~s}^{-2}$ (Antolin \& Verwichte 2011). Taking an average of $0.174 \mathrm{~km} \mathrm{~s}^{-2}$, the average velocity at the footpoint for our clumps would be around $150 \mathrm{~km} \mathrm{~s}^{-1}$ due to the effective gravity alone. From Bernouilli's Equation we can then estimate the increase of pressure due to the magnetic field downstream from the clump that would lead to a decrease of the final velocity of the clump (assuming no change in the gas pressure downstream of the clump). We obtain values of $0.3-0.5 \mathrm{dyn}^{-2}$ for average downstream plasma electron densities of $3-5 \times 10^{9} \mathrm{~cm}^{-3}$ (a factor of 10 lower than the average density derived from the EUV absorption measurement in Section 4.3, and consistent with numerical simulations, Antolin et al. 2010). These values correspond to a magnetic field increase of 2.8-3.6 G. On the other hand, the gas pressure created by the dense clump is $\approx 0.38 \mathrm{dyn}^{-2}$ (assuming a low ionization degree), matching the obtained values and therefore supporting this possibility.

Besides the interesting possibilities of coronal rain as local probes of the internal thermodynamic and magnetic loop structure, it can also serve as a global tracer of the magnetic field topology. This was first demonstrated in Paper I, where the angle of fall of rain clumps was calculated based on the full velocity vector, which allowed to trace the lower legs of loops and thus to distinguish several loop families. Here we show that the rain clumps' lengths may exist over a few tens of thousands of megameters, allowing further tracing of the loop legs. Such long rain structures have also been found recently by Scullion et al. (2014) in the context of post-flare loops, forming from the apex of loops. But even if short, by calculating the variance over relatively long periods of time as in Figure 14, or by detecting all the clumps crossing along perpendicular cuts to the trajectory at different heights as in Figure 5, the global shape of the loops' magnetic field can be inferred. By doing so we detect a clear expansion in the loops of data set 1 with CRISP. The width of the loop cross-section over which clumps are detected for loops 1 and 2 in Figure 5 decreases from 4." 6 to 2." 4 and 6 ". 8 to 3 ." 4 , respectively, from height $\mathrm{C}$ down to $\mathrm{F}$ separated over a distance of roughly $26^{\prime \prime}$ (similar to both loops). The width of the loops at the apex, detected with AIA in data sets 1 and 2 vary around $5^{\prime \prime}$, suggesting that the expansions we observe with CRISP are most of those experienced by these loops. On the other hand, in Paper I little expansion is observed, perhaps due to the difference in the height at which rain is detected. Here we detect it down to chromospheric heights, just before impact, while in the previous work only detection in the corona was possible. The expansion seems to occur mostly uniformly between heights $\mathrm{C}$ and $\mathrm{F}$, with the expansion occurring mostly above an observed kink in the trajectory of the clump, a little above height $\mathrm{F}$. In Figure 5, this kink is observed at $y \approx 35^{\prime \prime}$ (for loop 2 this kink is at a similar height). This kink for loops 1 and 2 is visible whenever a clump is observed and can be followed all the way down, suggesting that it is a feature of the magnetic field topology above the sunspot. Similar kinks in the field above sunspots have been detected by measuring the change with height of the dominant periods of running penumbral waves from the photosphere into the chromosphere (Jess et al. 2013).

\subsection{Persistent High-speed Downflows above Sunspots}

The chromospheric material observed along these thermally unstable loops occurs in clumps and appears intermittently at coronal heights. However, this downflow has a more persistent and constant character at chromospheric heights, just before impact. This is contrary to expected for the following reasons. At low chromospheric heights projection effects are stronger and visualization of clumps relies on absorption and therefore on high opacity. Unless the density of clumps is significant the resulting absorption would not be significant enough for allowing detection, which then rules out a significant number of clumps. Also, unless the filters have sufficiently narrow spectral bands the contribution from other chromospheric and photospheric emission will strongly reduce the contrast produced by the clumps. For these reasons, while off-limb detection of clumps can be performed in multiple wavelengths, on-disk detection is strongly limited. Even in the relatively high $\mathrm{H} \alpha$ opacity on-disk detection is usually strongly reduced to about $10 \%$ of the rain quantity, as shown in Antolin et al. (2012) and Paper I. Here, we show that under specific conditions of relatively high Doppler velocities from the $\mathrm{H} \alpha$ clumps with respect to the background (and therefore reduced projection effects in the wing of $\mathrm{H} \alpha$ ), detection of clumps just before impact is possible and the occurrence frequency of such downflows increases considerably, enough to give a persistent character.

The increase in coronal rain quantity at low heights may be the combination of two effects. The first effect is the continuous cooling, achieved in the case of complete thermal instability down to chromospheric temperatures. As discussed previously, in this study we obtain evidence for continuous cooling but in a strongly inhomogeneous way in which only small regions of the loops cool down catastrophically. Consequently, more rain in $\mathrm{H} \alpha$ will be observed at lower heights. The second effect may be more important, and is strongly dependent on the magnetic field geometry. The increase in coronal rain quantity may be caused by a funnel effect due to the increase of magnetic field strength at lower heights. As discussed in Section 5.3, here we observe a clear expansion of about a factor of two in radius for loops 1 and 2 from heights $\mathrm{F}$ to $\mathrm{C}$. On the other hand, only a slight change in the width of the clumps between both heights is found. This is shown in the upper panel of Figure 17, where the average of the clumps' widths in $\mathrm{H} \alpha$ for both loops changes from $0 . " 305$ to 0." 296 from cut $\mathrm{C}$ to $\mathrm{F}$ (shown in Figure 5), with nonetheless a significant decrease of the standard deviation from $0 . " 115$ to 0 ." 083 , respectively. From conservation of mass we would then expect both an increase in the frequency of rain crossing the cuts at chromospheric heights and, correspondingly, an increase of the clumps' lengths with decreasing height. Unfortunately, for the latter, we are unable to provide precise measurements of lengths at lower heights, due to the large projection effects and large speeds of the clumps (as shown in Figure 14 the height over which the clumps are significantly distinct from the background is limited to the low atmosphere and the region above the spicules). Although this tendency of elongation of clumps has been previously reported (Antolin et al. 2010, 2012; Antolin \& Rouppe van der Voort 2012) and is observed in general for data set 2 , this quantity is expected to be severely affected by other factors apart from the magnetic field expansion, such as flows, the effective gravity due to curvature, etc. This is reflected in the large scatter in lengths found for all instruments, shown in Figure 16. From this funnel effect one would also expect a decrease of the falling speeds from the compression of plasma downstream of the blob, as 
predicted by numerical simulations (Mackay \& Galsgaard 2001; Antolin et al. 2010). In our observations we could not detect a net decrease of falling speeds just before impact. However, it is possible that a velocity decrease is present but cannot be confidently detected due the elongation of clumps and the short distance above the chromosphere over which they offer enough contrast (which leads to a larger uncertainty in the measurements of projected speeds).

Persistent redshifts above sunspots have often been observed in TR and chromospheric lines (Brueckner 1981; Dere 1982; Kjeldseth-Moe et al. 1988; Brekke et al. 1990), often associated with bright fan-shaped structures observed in EUV above the umbra, usually termed plumes (Foukal et al. 1974; Brosius \& White 2004; Brosius 2005). The range of downflow speeds associated with such structures is large, mainly subsonic $\left(20-40 \mathrm{~km} \mathrm{~s}^{-1}\right)$, with supersonic second components of 100 $\mathrm{km} \mathrm{s}^{-1}$ or more (Brueckner 1981; Dere 1982; Nicolas et al. 1982; Kjeldseth-Moe et al. 1988; Brekke et al. 1990; Gurman 1993). Recently, similar events have been detected in TR lines with IRIS above the umbra of a sunspot, reaching speeds of 200 $\mathrm{km} \mathrm{s}^{-1}$ (Kleint et al. 2014). Such events, especially those presenting high-speed components, have been mainly related to either siphon flows (resulting from pressure imbalance at the footpoints) or condensations flows (issued from thermal instability in the coronal part of the loops). The latter scenario has been more frequently invoked over the years, especially when viewed in chromospheric lines, and since it can also generate siphon flows (Kleczek 1963; Kawaguchi 1970; Leroy 1972; Foukal 1978; Makhmudov et al. 1980; de Groof et al. 2004; Müller et al. 2005; Antolin et al. 2012), also supported by numerical simulations (Goldsmith 1971; Hildner 1974; Mok et al. 1990; Reale et al. 1996, 1997; Müller et al. 2003, 2004, 2005; Mendoza-Briceño et al. 2005; Mok et al. 2008; Antolin et al. 2010; Xia et al. 2011; Fang et al. 2013).

At high resolution, especially in chromospheric lines, coronal rain downflows have been shown to have a clumpy character, thus suggesting a rather intermittent and bursty downflow character, even if frequent, at odds with some observations of persistent and continuous downflows above sunspots. The present observations suggest that the intermittent and clumpy character of cool coronal rain (observed in chromospheric lines) can become persistent and continuous at chromospheric heights, thereby providing an answer to the long standing problem of downflows. When viewed in warmer TR lines this character may be further enhanced. Indeed, we find that in general the widths of the clumps increase in TR lines, which may lead to the same effect. In AIA the darkening of the EUV loops is observed to have a persistent and continuous character. The persistency of the rain recently reported in Kleint et al. (2014) also supports this scenario.

\subsection{Role in the Chromosphere-corona Mass Cycle}

Here we show that clumps are multithermal, suggesting a highly inhomogeneous density structure. Although a thorough statistical study of densities of coronal rain clumps is needed, we can make a rough estimate of the mass density flux involved in the current process for data set 1 . For this we assume that the mass of each condensation is roughly proportional to its length, a result supported by 3D numerical calculations (Luna et al. 2012). The electron density for a particular clump is estimated to vary between $2 \times 10^{10}$ and $2.5 \times 10^{11} \mathrm{~cm}^{-3}$.
Taking an average of $1.4 \times 10^{11} \mathrm{~cm}^{-3}$ for all $\mathrm{H} \alpha$ clumps, an average width of $0 . " 4$, and an average length of $3 . " 8$, and taking into account that the total number of $\mathrm{H} \alpha$ clumps is 479 for the two loops over a time of $95 \mathrm{~min}$, we obtain an average downward mass flux for one loop of $1.23 \times 10^{9} \mathrm{~g} \mathrm{~s}^{-1}$. For data set 2 , assuming a similar density and using the corresponding values found for that data set (see Table 1), we find a value of $5.22 \times 10^{9} \mathrm{~g} \mathrm{~s}^{-1}$. These values are on the same order as that estimated in Paper I, where, the observed active region comprised multiple rain loops (five or six loops can be distiguished, as shown in their Figures 14 and 17) and a corresponding higher number of clumps (the assumed average rain density in that paper was however assumed 2.5 times lower than that estimated here). These are significant values comparable to those found for prominence drainage (Berger et al. 2012; Liu et al. 2012). This agreement over different data sets strongly suggests an important role of coronal rain in the chromosphere-corona mass cycle.

As discussed in Section 5.3, the detected number of clumps in $\mathrm{H} \alpha$ may very well just be the tip of the iceberg, and many more unresolved clumps may exist. Let's assume nonetheless that we detect most of the rain in both loops over the observed time sequence, which would then correspond to the amount of material in these loops involved in one limit cycle (assuming that most of the material in one loop is thermally unstable). We can then calculate the average density in these loops. For this we assume the loop to be a straight cylinder with an average width of 5."7 (the average between both loops of the transverse distance over which clumps are detected at heights $C$ ) and a loop length of $130 \mathrm{Mm}$, as estimated in Section 3.2 (assuming a similar length for both loops). We obtain an average electron density of $6.2 \times 10^{8} \mathrm{~cm}^{-3}$. Using corresponding values for the loop in data set 2 a value of $7.3 \times 10^{8} \mathrm{~cm}^{-3}$ is obtained. These values are at least five times below the expected density of active region loops in a thermal non-equilibrium state, obtained from numerical simulations. This suggests three possibilities. In the first and the second, the loop does not become fully thermally unstable with only parts of it condensing and falling as coronal rain. In the first scenario, most of the loop's mass remains at coronal temperatures and undergoes a different thermodynamical evolution. In the second, the entire loop cools but only a small part of it reaches chromospheric temperatures while most of it stays at TR temperatures. The third scenario consists of a fully thermally unstable loop, with most of the mass reaching chromospheric temperatures but involving rain clumps smaller than those presently resolved. The first scenario would imply that neighboring strands do not generally cool down at the same time, something at odds with previous (Paper I) and the present results (especially for data set 1, while for data set 2 it is possible that a significant amount of material at coronal temperatures is still present). In the second scenario, we would expect the bulk of the rain distribution to consist of less dense and warmer clumps being larger and longer (for instance, three times larger and longer clumps and twice the currently detected number would suffice). While this roughly matches the AIA statistics for data set 1 , it is however at odds with the IRIS results of data set 2, which show a very similar morphology and distribution of clumps in chromospheric and TR temperatures, and especially far fewer number of clumps at high temperatures. Hence, the most likely possibility is the third, and involves a far larger number of unresolved chromospheric clumps. This result suggests that the main contributor 
to downflows (in terms of mass) in thermally unstable loops is coronal rain. This scenario should be tested in 3D numerical simulations.

\section{CONCLUSIONS}

In this paper two data sets that combine multi-wavelength observations with SST/CRISP, Hinode/SOT, IRIS/SJI, and SDO/AIA, spanning chromospheric, TR, and coronal temperatures, are used to reveal the multi-strand and multithermal aspects of coronal rain at unprecedented detail. We provide important characteristics of the thermally unstable plasma ending up as coronal rain, and more generally of the thermal instability mechanism in the corona. We show how this mechanism can play a significant role into the shaping of coronal structures. Furthermore, we take the first steps toward estimating the fraction of coronal plasma in a normal active region undergoing full catastrophic cooling. The results can be listed as follows.

1. While the standard model of progressive cooling is observed, in which light curves peak in order of decreasing temperature, we also find a case in which coronal emission is present and is even enhanced cotemporally and co-spatially with the chromospheric and TR emission from coronal rain. Loops in a thermal nonequilibrium state may therefore not forcibly show gradual systematic cooling from coronal to chromospheric temperatures, i.e., a sequential EUV intensity variation from hot to cool temperatures (where the hot emission is significantly reduced) is not a necessary observational condition of thermally unstable loops.

2. EUV intensity darkening is found strongly correlated in space to coronal rain appearance. Furthermore, the rain can produce EUV intensity variations on short timescales of a few minutes that can appear quasi-periodic over time.

3. Progressive cooling of coronal rain is observed, leading to a height dependence of the emission. Catastrophic cooling is observed to follow two stages: a rapid cooling throughout TR temperatures and a slower cooling down to chromospheric temperatures, which may reflect the transition to optically thick plasma states.

4. Coronal rain is a highly multithermal phenomenon encompassing TR and chromospheric temperatures. We find a high degree of co-spatiality in the multi-wavelength emission implying a strong degree of density inhomogeneity within thermally unstable loops. A thin transition from chromospheric to TR temperatures must exist on spatial scales lower than 0." 33 .

5. We estimate coronal rain core electron densities to vary between $2 \times 10^{10}$ and $2.5 \times 10^{11} \mathrm{~cm}^{-3}$.

6. The distribution of coronal rain widths is found to be rather independent of temperature with a clear increase of clump number (and a decrease of standard deviation) at small scales for each wavelength. The number of clumps is, however, found to increase sharply across the temperatures toward the chromospheric range, reinforcing the tip-of-the-iceberg scenario proposed in Paper I. The present results strongly suggest that the bulk of the distribution is below the highest resolution used here $(\approx 0 . " 2)$.

7. The coronal rain clumps appear organized in strands. Such structure is not limited to chromospheric temperatures but extends at least to transition temperatures as well, suggesting an important role of thermal instability in the shaping of fundamental loop substructure. At the smallest detected scales we find structure reminiscent of the MHD thermal mode (Field 1965; van der Linden \& Goossens 1991a, 1991b; Murawski et al. 2011).

8. Through order-of-magnitude estimates we show that the strong density inhomogeneity that we find entails a local increase of gas pressure accompanied by a non-local increase of magnetic pressure due to flux freezing all along the clump's trajectory (up- and downstream), thereby shaping hotter material into strands. We further suggest that this mechanism may explain the commonly observed lower than freefall speeds of coronal rain.

9. We show how coronal rain can serve as a global tracer of the magnetic field topology. We detect the presence of a clear kink in the field in the upper chromosphere above sunspots, reminiscent of the sharp change in angle reported in Jess et al. (2013) in a similar location. The magnetic field is found to expand roughly uniformly above this kink up to coronal heights over at least a factor of two in radius.

10. The intermittent and clumpy appearance of coronal rain at coronal heights becomes more continuous and persistent at chromospheric heights just before impact. This is likely due to progressive cooling of the rain and, especially, to a funnel effect from the expansion of the magnetic field at low heights.

11. The persistent (and burst-less) character of coronal rain at low heights is in agreement with the common detection of moderate and high-speed redshifts above sunspots in chromospheric and TR lines, and may thus offer an explanation to this long standing problem.

12. In terms of filling factor (and therefore detectability, especially with poor resolution instruments), coronal rain is mainly a TR phenomenon. However, in terms of mass, the chromospheric component of the cooling plasma plays a major role in the mass cycle of the loop. We find average downward mass fluxes per loop of $1-5 \times$ $10^{9} \mathrm{~g} \mathrm{~s}^{-1}$, in accordance with previous estimates in Paper I, and prominence mass drainage values (Berger et al. 2012; Liu et al. 2012).

P. A. would like to thank W. Liu, B. C. Low, B. De Pontieu, R. Soler and the anonymous referee for fruitful comments and discussions that led to a significant improvement of the manuscript. P. A. acknowledges support from the International Space Science Institute, Bern, Switzerland to the International Team on "Implications for coronal heating and magnetic fields from coronal rain observations and modeling." This research was supported by Japan Society for the Promotion of Science (JSPS) of Grant-in-Aid for Scientific Research (A) (Grant Number 25220703, PI: S. Tsuneta). G. V., T. P., and L. R. acknowledge funding by the Norwegian Research Council, and G. V., T. P. also by the European Research Council under the European Union's Seventh Framework Programme (FP7/ 2007-2013)/ERC grant agreement nr.291058. E.S. is a Government of Ireland Post-doctoral Research Fellow supported by the Irish Research Council (IRC). IRIS is a NASA small explorer mission developed and operated by LMSAL with mission operations executed at NASA Ames Research 
Center and major contributions to downlink communications funded by the Norwegian Space Center through an ESA PRODEX contract. The SST is operated on the island of La Palma by the Institute for Solar Physics of Stockholm University in the Spanish Observatorio del Roque de los Muchachos of the Instituto de Astrofísica de Canarias. Hinode is a Japanese mission developed and launched by ISAS/JAXA, with NAOJ as domestic partner and NASA and STFC (UK) as international partners. It is operated by these agencies in cooperation with ESA and NSC (Norway). SDO is part of NASA's Living With a Star Program. This work was (partly) carried out on the Solar Data Analysis System operated by the Astronomy Data Center in cooperation with the Hinode Science Center of the National Astronomical Observatory of Japan.

\section{REFERENCES}

Ahn, K., Chae, J., Cho, K.-S., et al. 2014, SoPh, 289, 4117

An, C.-H. 1984, ApJ, 276, 755

Antiochos, S. K., MacNeice, P. J., Spicer, D. S., \& Klimchuk, J. A. 1999, ApJ, 512, 985

Antolin, P., \& Rouppe van der Voort, L. 2012, ApJ, 745, 152

Antolin, P., Shibata, K., \& Vissers, G. 2010, ApJ, 716, 154

Antolin, P., \& Verwichte, E. 2011, ApJ, 736, 121

Antolin, P., Vissers, G., \& Rouppe van der Voort, L. 2012, SoPh, 280, 457

Anzer, U., \& Heinzel, P. 2005, ApJ, 622, 714

Aschwanden, M. J., Schrijver, C. J., \& Alexander, D. 2001, ApJ, 550, 1036

Ayres, T., \& France, K. 2010, ApJL, 723, L38

Berger, T. E., Liu, W., \& Low, B. C. 2012, ApJL, 758, L37

Brekke, P., Kjeldseth-Moe, O., \& Brueckner, G. E. 1990, Ap\&SS, 170, 135

Brooks, D. H., Warren, H. P., \& Ugarte-Urra, I. 2012, ApJL, 755, L33

Brooks, D. H., Warren, H. P., Ugarte-Urra, I., \& Winebarger, A. R. 2013, ApJL, 772, L19

Brosius, J. W. 2005, ApJ, 622, 1216

Brosius, J. W., \& White, S. M. 2004, ApJ, 601, 546

Brueckner, G. E. 1981, in Solar Active Regions: A monograph from Skylab Solar Workshop III, ed. F. Q. Orrall (Boulder, CO: Colorado Associated Univ. Press), 113

Chiuderi, C., \& Chiuderi Drago, F. 1991, SoPh, 132, 81

De Groof, A., Berghmans, D., van Driel-Gesztelyi, L., \& Poedts, S. 2004, A\&A, 415, 1141

de la Cruz Rodríguez, J., Löfdahl, M. G., Sütterlin, P., Hillberg, T., \& Rouppe van der Voort, L. 2015, A\&A, 573, A40

De Pontieu, B., Title, A. M., Lemen, J. R., et al. 2014, SoPh, 289, 2733

Dere, K. P. 1982, SoPh, 77, 77

Fang, X., Xia, C., \& Keppens, R. 2013, ApJL, 771, L29

Field, G. B. 1965, ApJ, 142, 531

Foukal, P. 1978, ApJ, 223, 1046

Foukal, P. V. 1976, ApJ, 210, 575

Foukal, P. V., Noyes, R. W., Reeves, E. M., et al. 1974, ApJL, 193, L143

Froment, C., Auchére, F., Bocchialini, K., et al. 2015, arXiv:1504.08129

Goedbloed, J. P. H., \& Poedts, S. 2004, Principles of Magnetohydrodynamics (Cambridge: Cambridge Univ. Press)

Goldsmith, D. W. 1971, SoPh, 19, 86

Gurman, J. B. 1993, ApJ, 412, 865

Harra, L. K., Matthews, S. A., Long, D. M., Doschek, G. A., \& De Pontieu, B. 2014, ApJ, 792, 93

Heinzel, P., \& Anzer, U. 2006, ApJL, 643, L65

Heinzel, P., Schmieder, B., Vial, J.-C., \& Kotrč, P. 2001, A\&A, 370, 281

Hildner, E. 1974, SoPh, 35, 123

Jess, D. B., Reznikova, V. E., van Doorsselaere, T., Keys, P. H., \& Mackay, D. H. 2013, ApJ, 779, 168

Kamio, S., Peter, H., Curdt, W., \& Solanki, S. K. 2011, A\&A, 532, A96

Karpen, J. T., Antiochos, S. K., Hohensee, M., Klimchuk, J. A., \& MacNeice, P. J. 2001, ApJL, 553, L85

Kawaguchi, I. 1970, PASJ, 22, 405

Kjeldseth-Moe, O., \& Brekke, P. 1998, SoPh, 182, 73

Kjeldseth-Moe, O., Brynildsen, N., Brekke, P., et al. 1988, ApJ, 334, 1066

Kleczek, F. 1963, BAICz, 14, 167
Kleint, L., Antolin, P., Tian, H., et al. 2014, ApJL, 789, L42

Klimchuk, J. A. 2006, SoPh, 234, 41

Klimchuk, J. A. 2014, in Proc. of Joint AAS/AGU Triennial Earth-Sun Summit, 1, 203.08

Klimchuk, J. A., Karpen, J. T., \& Antiochos, S. K. 2010, ApJ, 714, 1239

Labrosse, N., Heinzel, P., Vial, J.-C., et al. 2010, SSRv, 151, 243

Landi, E., Miralles, M. P., Curdt, W., \& Hara, H. 2009, ApJ, 695, 221

Landi, E., \& Reale, F. 2013, ApJ, 772, 71

Lemen, J. R., Title, A. M., Akin, D. J., et al. 2011, SoPh, 172

Leroy, J. 1972, SoPh, 25, 413

Lin, Y., Engvold, O., Rouppe van der Voort, L., Wiik, J. E., \& Berger, T. E. 2005, SoPh, 226, 239

Lionello, R., Winebarger, A. R., Mok, Y., Linker, J. A., \& Mikić, Z. 2013, ApJ, 773, 134

Liu, W., Berger, T. E., \& Low, B. C. 2012, ApJL, 745, L21

Low, B. C., Berger, T., Casini, R., \& Liu, W. 2012a, ApJ, 755, 34

Low, B. C., Liu, W., Berger, T., \& Casini, R. 2012b, ApJ, 757, 21

Luna, M., Karpen, J. T., \& DeVore, C. R. 2012, ApJ, 746, 30

Mackay, D. H., \& Galsgaard, K. 2001, SoPh, 198, 289

Makhmudov, M. M., Nikolskii, G. M., \& Zhugzhda, I. D. 1980, SoPh, 66, 89

Mendoza-Briceño, C. A., Sigalotti, L. D. G., \& Erdélyi, R. 2005, ApJ, 624,1080

Mikić, Z., Lionello, R., Mok, Y., Linker, J. A., \& Winebarger, A. R. 2013, ApJ, 773, 94

Mok, Y., Drake, J. F., Schnack, D. D., \& van Hoven, G. 1990, ApJ, 359, 228

Mok, Y., Mikić, Z., Lionello, R., \& Linker, J. A. 2008, ApJL, 679, L161

Müller, D. A. N., De Groof, A., Hansteen, V. H., \& Peter, H. 2005, A\&A, 436, 1067

Müller, D. A. N., Hansteen, V. H., \& Peter, H. 2003, A\&A, 411, 605

Müller, D. A. N., Peter, H., \& Hansteen, V. H. 2004, A\&A, 424, 289

Murawski, K., Zaqarashvili, T. V., \& Nakariakov, V. M. 2011, A\&A, 533, A18

Nicolas, K. R., Bartoe, J.-D. F., Brueckner, G. E., \& Kjeldseth-Moe, O. 1982, SoPh, 81, 253

Okamoto, T. J., Tsuneta, S., Berger, T. E., et al. 2007, Sci, 318, 1577

O'Shea, E., Banerjee, D., \& Doyle, J. G. 2007, A\&A, 475, L25

Parenti, S. 2015, in Astrophysics and Space Science Library, Vol. 415 ed. J.-C. Vial, \& O. Engvold (Berlin: Springer), 61

Peter, H., Bingert, S., \& Kamio, S. 2012, A\&A, 537, A152

Peter, H., Bingert, S., Klimchuk, J. A., et al. 2013, A\&A, 556, A104

Reale, F. 2010, LRSP, 7, 5

Reale, F., Orlando, S., Testa, P., Landi, E., \& Schrijver, C. J. 2014, ApJL, 797, L5

Reale, F., Orlando, S., Testa, P., et al. 2013, Sci, 341, 251

Reale, F., Peres, G., \& Serio, S. 1996, A\&A, 316, 215

Reale, F., Peres, G., \& Serio, S. 1997, A\&A, 318, 506

Scharmer, G. B., Bjelksjo, K., Korhonen, T. K., Lindberg, B., \& Petterson, B. 2003a, in SPIE Conf. Ser. 4853, ed. S. L. Keil, \& S. V. Avakyan, 341

Scharmer, G. B., Dettori, P. M., Lofdahl, M. G., \& Shand, M. 2003b, Proc. SPIE, 4853, 370

Scharmer, G. B., Narayan, G., Hillberg, T., et al. 2008, ApJL, 689, L69

Schrijver, C. J. 2001, SoPh, 198, 325

Scullion, E., Rouppe van der Voort, L., Wedemeyer, S., \& Antolin, P. 2014, ApJ, 797, 36

Secchi, A. 1875, Le Soleil (Paris: Gauthier-Villars), 422

Sharma, P., Parrish, I. J., \& Quataert, E. 2010, ApJ, 720, 652

Soler, R., Ballester, J. L., \& Parenti, S. 2012, A\&A, 540, A7

Susino, R., Lanzafame, A. C., Lanza, A. F., \& Spadaro, D. 2010, ApJ, 709, 499

Tripathi, D., Mason, H. E., Dwivedi, B. N., del Zanna, G., \& Young, P. R. 2009, ApJ, 694, 1256

Tsuneta, S., Ichimoto, K., Katsukawa, Y., et al. 2008, SoPh, 249, 167

Ugarte-Urra, I., Warren, H. P., \& Brooks, D. H. 2009, ApJ, 695, 642

Ugarte-Urra, I., Winebarger, A. R., \& Warren, H. P. 2006, ApJ, 643, 1245

van der Linden, R. A. M., \& Goossens, M. 1991a, SoPh, 134, 247

van der Linden, R. A. M., \& Goossens, M. 1991b, SoPh, 131, 79

van Noort, M., Rouppe van der Voort, L., \& Löfdahl, M. G. 2005, SoPh, 228, 191

Vial, J.-C., Olivier, K., Philippon, A. A., Vourlidas, A., \& Yurchyshyn, V. 2012, A\&A, 541, A108

Viall, N. M., \& Klimchuk, J. A. 2012, ApJ, 753, 35

Vissers, G., \& Rouppe van der Voort, L. 2012, ApJ, 750, 22

Warren, H. P., Ugarte-Urra, I., Brooks, D. H., et al. 2007, PASJ, 59, 675

Xia, C., Chen, P. F., Keppens, R., \& van Marle, A. J. 2011, ApJ, 737, 27 